\title{
Transit timing variations in eccentric hierarchical triple exoplanetary systems
}

\section{Perturbations on the time scale of the orbital period of the perturber}

\author{
T. Borkovits ${ }^{1}$, Sz. Csizmadia ${ }^{2}$, E. Forgács-Dajka ${ }^{3}$, and T. Hegedüs ${ }^{1}$ \\ 1 Baja Astronomical Observatory of Bács-Kiskun County, 6500 Baja, Szegedi út, Kt. 766, Hungary \\ e-mail: [borko; hege]@electra.bajaobs.hu \\ ${ }^{2}$ Deutsches Zentrum für Luft- und Raumfahrt (DLR), Institut für Planetenforschung, Rutherfordstr. 2, 12489 Berlin, Germany \\ e-mail: szilard.csizmadia@dlr.de \\ 3 Eötvös University, Department of Astronomy, 1518 Budapest, Pf. 32, Hungary \\ e-mail: E.Forgacs-Dajka@astro.elte.hu
}

Received 5 October 2010 / Accepted 16 December 2010

\section{ABSTRACT}

\begin{abstract}
Aims. We study the long-term time scale (i.e. period comparable to the orbital period of the outer perturber object) transit timing variations (TTVs) in transiting exoplanetary systems that contain another more distant $\left(a_{2} \gg a_{1}\right)$ planetary or stellar companion. Methods. We give an analytical form of the $\mathrm{O}-\mathrm{C}$ diagram (which describes such TTVs) in a trigonometric series, which is valid for arbitrary mutual inclinations, up to the sixth order in the inner eccentricity.

Results. We show that the dependence of the $\mathrm{O}-\mathrm{C}$ on the orbital and physical parameters can be separated into three parts. Two of these are independent of the real physical parameters (i.e. masses, separations, periods) of a concrete system and only depend on dimensionless orbital elements, so can be analysed in general. We find that, for a specific transiting system, where eccentricity $\left(e_{1}\right)$ and the observable argument of periastron $\left(\omega_{1}\right)$ are known, say, from spectroscopy, the main characteristics of that TTV, which is caused by a possible third-body can be mapped simply. Moreover, as the physical attributes of a given system only occur as scaling parameters, the real amplitude of the $\mathrm{O}-\mathrm{C}$ can also be estimated for a given system, simply as a function of the $m_{3} / P_{2}$ ratio. We analyse the above-mentioned dimensionless amplitudes for different arbitrary initial parameters, as well as for two particular systems, CoRoT-9b and HD 80606b. We find in general that, while the shape of the O-C strongly varies with the angular orbital elements, the net amplitude (departing from some specific configurations) depends only weakly on these elements, but strongly on the eccentricities. As an application, we illustrate how the formulae work for the weakly eccentric CoRoT-9b and the highly eccentric HD 80606b. We also consider the question of detection, as well as the correct identification of such perturbations. Finally, we illustrate the operation and effectiveness of Kozai cycles with tidal friction (KCTF) in the case of HD 80606b.
\end{abstract}

Key words. methods: analytical - planets and satellites: individual: CoRoT-9b - planets and satellites: individual: HD 80606b methods: numerical - planetary systems - binaries: close

\section{Introduction}

The rapidly increasing number of exoplanetary systems, as well as the lengthening time interval of the observations naturally leads to the search for perturbations in the motion of the known planets, and this can provide the possibility of detecting additional planetary (or stellar) components in a given system, can produce further information about the oblateness of the host star (or the planet), or might even refer to evolutionary effects.

The detection and the interpretation of such perturbations in the orbital revolution of the exoplanets usually depends on such methods and theoretical formulae, which are well known and have been applied for a long time in the field of the close eclipsing and spectroscopic binaries. We mainly refer to the methods developed in connection with the observed period variations in eclipsing binaries. These period variations manifest themselves in non-linear variations of the difference between the observed and the predicted time of mid-eclipses (either transits or occultations). In the nomenclature of exoplanet studies, this phenomenon is called transit timing variation (TTV). Plotting the observed minus the calculated mid-transit times with respect to the cycle numbers, we get the $\mathrm{O}-\mathrm{C}$ diagram, which has been the main tool for period studies by observers of variable stars (not only for eclipsing binaries) for more than a century. Consequently, the effect of the various types of period variations (both real and apparent) for the $\mathrm{O}-\mathrm{C}$ diagram have already been widely studied in the past one hundred years. Some of these are less relevant in the case of transiting exoplanets, but others are important. For example, the two classical cases are the simple geometrical light-time effect (LITE) (of another, more distant companion), and the apsidal motion effect (AME) (of both the stellar oblateness in eccentric binaries and the relativistic effect). LITE has been widely used for identifying other stellar companions of many variable stars (not only eclipsing binaries) from the pioneering papers of Chandler (1892), Hertzsprung (1922), Woltjer (1922), and Irwin (1952) to a copious number of recent applications. Owing to its small amplitude, however, it is less significant in the case of a planetarymass wide component. Nevertheless in recent years, there have been some efforts to discover exoplanets in this manner (see e.g. Silvotti et al. 2007, V391 Pegasi; Deeg et al. 2008, CM Draconis; Qian et al. 2009, QS Virginis; Lee et al. 2009, HW Virginis). The importance of AME in close exoplanet systems has been investigated in several papers; see e.g. Miralda-Escudé (2002), 
Heyl \& Gladman (2007), and Jordán \& Bakos (2008, and further references thereins). This effect has been studied since the pioneering works of Cowling (1938), and Sterne (1939), and besides its evident importance in the checking of the general relativity theory, it plays also an important role in studies of the inner mass distributions of stars (via their quadrupol moment); i.e. it provides observational verification of stellar models.

Besides their similarities, there are also several differences between the period variations of close binary and multiple stars, and of planetary systems. For example, most of the known stellar multiple systems form hierarchic subsystems (see e.g. Tokovinin 1997). This is mainly the consequence of the dynamical stability of these configurations, or put another way, the dynamical instability of the non-hierarchical stellar multiple systems. In contrast, a multiple planetary system may form a stable (or at least long-time quasi-stable) non-hierarchical configuration, as we can see in our solar system, and furthermore, the same result has been shown by numeric integrations for several known exoplanet systems (see e.g. Sándor et al. 2007). This means we can expect several different configurations, the examination of which has, until now, not been considered in the field of the period variations of multiple stellar systems. Examples of these phenomena are the perturbations of both an inner planet and a companion on a resonant orbit (Agol et al. 2005) or, as a special case of the latter, the possibility of Trojan exoplanets (Schwarz et al. 2009). Recently, the detectability of exomoons has also been studied (Simon et al. 2007; Kipping 2009a,b).

Furthermore, thanks to the enhanced activities related to extrasolar planetary searches, which led to missions like CoRoT and Kepler that produce long-term, extraordinarily accurate data, we can expect in the near future other such observations to make it possible to detect and study more phenomena never observed earlier. For example, it is well known that neither the close binary stars nor the hot-Jupiter-type exoplanets could have been formed in their present positions. Different orbital shrinking mechanisms are described in the literature. Instead of listing them, we refer to the short summaries by Tokovinin et al. (2006), Tokovinin (2008), and Fabrycky \& Tremaine (2007). Here we note only that one of the most preferred theories for the formation of close binary stars, which also might have produced at least a portion of hot Jupiters, as well, is the combination of the Kozai cycles with tidal friction (KCTF). The Kozai resonance (recently and frequently referred to as Kozai cycle[s]) was first described by Kozai (1962) when investigating secular perturbations of asteroids. The first (theoretical) investigation of this phenomenon with respect to multiple stellar systems can be found in the studies by Harrington (1968, 1969), Mazeh \& Saham (1979), and Söderhjelm (1982). A higher, third-order theory of Kozai cycles was given by Ford et al. (2000), while the first application of KCTF to explain the present configuration of a close, hierarchical triple system (the emblematic Algol, itself) was presented by Kiseleva et al. (1998). According to this theory, the close binaries (as well as hot-Jupiter systems) should have originally formed as significantly wider primordial binaries having a distant, inclined third companion. Due to the third object's induced Kozai oscillation, the inner eccentricity becomes cyclically so large, that around the periastron passages, the two stars (or the host star and its planet) approach each other so closely that tidal friction may be effective, which shrinks the orbit remarkably, during one or more Kozai cycles. Owing to the smaller separation, the tidal forces remain effective on a larger and larger portion of the whole revolution, and finally, they will switch off the Kozai cycles, producing a highly eccentric, moderately inclined, small-separation intermediate orbit. After the last
Kozai cycle, some additional, tidally forced circularization may then form close systems in their recent configurations.

Nevertheless, independently of the question, as to which mechanism(s) is (are) the really effective ones, up to now we have not been able to study these mechanisms in operation, so only the end-results have been observed. This is mainly the consequence of selection effects. In order to study these phenomena when they are effective, one should observe the variation in the orbital elements of such extrasolar planets, as well as binary systems that are in the period range of months to years. The easisest way to carry out such observations is to monitor the TTVs of these systems. However, there are only a few known transiting extrasolar planets in this period regime. Furthermore, although binary stars are also known with such separations, they are not appropriate subjects for this investigation because of their noneclipsing nature.

The continuous long-term monitoring of several hundred stars with the CoRoT and Kepler satellites, as well as the long-term systematic terrestrial surveys, provide an excellent opportunity to discover transiting exoplanets (or as by-products: eclipsing binaries) with the period of months. Then continuous, long-term transit monitoring of these systems (combining the data with spectroscopy) may allow dynamical evolutionary effects (i.e. orbital shrinking) to already be traced within a few decades. Furthermore, the larger the characteristic size of a multiple planetary, stellar (or mixed) system the greater the amplitude of even the shorter period perturbations in the TTVs, as shown in detail in the discussion of Borkovits et al. (2003).

In the past few years, several papers have been published on TTVs, both from theoretical aspects (e.g. Agol et al. 2005; Holman \& Murray 2005; Nesvorný \& Beaugé 2010; Holman 2010; Cabrera 2010; Fabrycky 2010, see further references therein) and from large numbers of papers on observational aspects for individual transiting exoplanetary systems. Nevertheless, most (but not all) of the theoretical papers above mainly concentrate on the detectibility of additional companions (especially super-Earths) from the TTVs.

In this paper we consider this question in greater detail. We calculate the analytical form of the long-period ${ }^{1}$ (i.e. with a period close to the orbital period of the ternary component $P_{2}$ ), time-scale perturbations of the $\mathrm{O}-\mathrm{C}$ diagram for hierarchical (i.e. $P_{2} \gg P_{1}$ ) triple systems. (As we mainly concentrated on transiting systems with a period of weeks to months, we omitted the possible tidal forces. However, our formulae can be practically applied even for the closest exoplanetary systems, because the tidal perturbations usually become effective on a notably

${ }^{1}$ In this paper we follow the original classification of Brown (1936), which divided the periodic perturbations occurring in hierarchical systems into the following three groups:

\footnotetext{
- short-period perturbations. The typical period is equal to the orbital period $P_{1}$ of the close pair, while the amplitude has the order $\left(P_{1} / P_{2}\right)^{2}$;

- long-period perturbations. This group has a typical period of $P_{2}$, and magnitude of the order $\left(P_{1} / P_{2}\right)$;

- apse-node terms. In this group the typical period is about $P_{2}^{2} / P_{1}$, and the order of the amplitude reaches unity.
}

This classification differs from what is used in the classical planetary perturbation theories. There, the first two groups are termed together as "short period" perturbations, while the "apse-node terms" are called "long period ones". Nevertheless, in the hierarchical scenario, the first two groups differ from each other both in period and amplitude; consequently, we feel this nomenclature is more appropriate in the present situation. 
T. Borkovits et al.: Transit timing variations in eccentric hierarchical triple exoplanetary systems. I.

longer time scale.) This work is a continuation and extension of the previous paper of Borkovits et al. (2003). In that paper we formulated the long-period perturbations of an (arbitrarily eccentric and inclined) distant companion to the $\mathrm{O}-\mathrm{C}$ diagram for a circular inner orbit. (Our formula is more general than that of Agol et al. 2005. For the coplanar case the two results become identical.) Now we extend the results to the case of an eccentric inner binary (formed either a host star with its planet or two stars). As shown, our formulae have a satisfactory accuracy even for high eccentricity, such as $e_{1}=0.9$. In the period regime of a few months, the tidal forces are ineffective, so we expect eccentric orbits. This is especially valid in the case of the predecessor systems of hot Jupiters, in which case the formation theory mentioned above predicts very high eccentricities.

In the next section we give a very brief summary of our calculations. (A somewhat detailed description can be found in Borkovits et al. 2003, 2007; nevertheless for self-consistency of the present paper we provide a brief overview.) In Sect. 3 we discuss our results, while in Sect. 4 we illustrate the results with both analytical and numerical calculations on two individual systems, CoRoT-9b and HD 80606b. Finally in Sect. 5 we draw conclusions from our results, and, furthermore, we compare our method and results with those of Nesvorný \& Morbidelli (2008), and Nesvorný (2009).

\section{Analytical investigations}

\subsection{General considerations and equations of the problem}

As is well known, at the moment of the mid-transit (which for an eccentric orbit usually does not coincide with half the whole transit event)

$u \approx \pm \frac{\pi}{2}+2 k \pi$

where $u$ is the true longitude measured from the intersection of the orbital plane and the plane of sky, and $k$ is an integer. (Since, traditionally the positive $z$-axis is directed away from the observer, the primary transit occurs at $u=-\pi / 2$.) An exact equality is valid only if the binary has a circular orbit, or if the orbit is seen edge-on exactly. The correct inclination dependence of the occurrence of the mid-eclipses can be found in Gimènez \& Garcia-Pelayo (1983). Nevertheless, the observable inclination of a potentially month-long period transiting extrasolar planet should be close to $i=90^{\circ}$, if the latter condition is to be satisfied. Due to its key role in the transits we use $u$ as our independent time-like variable, instead of the usual variables. It is known from the textbooks of celestial mechanics that

$$
\begin{aligned}
\dot{u} & =\frac{c_{1}}{\rho_{1}^{2}}-\dot{\Omega} \cos i, \\
& =\mu^{1 / 2} a^{-3 / 2}\left(1-e^{2}\right)^{-3 / 2}(1+e \cos v)^{2}-\dot{\Omega} \cos i ;
\end{aligned}
$$

consequently, the moment of the $N$ th primary minimum (transit) after an epoch $t_{0}$ can be calculated as

$$
\begin{aligned}
\int_{t_{0}}^{t_{N}} \mathrm{~d} t & =\int_{-\pi / 2}^{2 N \pi-\pi / 2} \frac{a^{3 / 2}}{\mu^{1 / 2}} \frac{\left(1-e^{2}\right)^{3 / 2}}{[1+e \cos (u-\omega)]^{2}} \frac{\mathrm{d} u}{1-\frac{\rho_{1}^{2}}{c_{1}} \dot{\Omega} \cos i}, \\
& \approx \int \frac{a^{3 / 2}}{\mu^{1 / 2}} \frac{\left(1-e^{2}\right)^{3 / 2}}{[1+e \cos (u-\omega)]^{2}}\left(1+\frac{\rho_{1}^{2}}{c_{1}} \dot{\Omega} \cos i\right) \mathrm{d} u
\end{aligned}
$$

In the equations above, $c_{1}$ denotes the specific angular momentum of the inner binary, $\rho_{1}$ is the radius vector of the planet with respect to its host star, and the orbital elements have their usual meanings. Furthermore, in Eq. (3) we write the true anomaly as $v=u-\omega$. To evaluate Eq. (3) we first have to express the perturbations in the orbital elements with respect to $u$. Assuming that the orbital elements (except of $u$ ) are constant, the first term of the right-hand side (r.h.s.) yields the following closed solution

$\bar{P}_{\mathrm{I}, \mathrm{II}}=\frac{P}{2 \pi}\left[2 \arctan \left(\sqrt{\frac{1-e}{1+e}} \frac{\mp \cos \omega}{1 \mp \sin \omega}\right) \pm\left(1-e^{2}\right)^{1 / 2} \frac{e \cos \omega}{1 \mp e \sin \omega}\right]$,

for the two types of minima, respectively. (Here $P$ denotes the anomalistic or Keplerian period which is considered to be constant.) Instead of the exact forms above, widely used is the expansion (as in this paper), which, up to the fifth order in $e$, is

$$
\begin{aligned}
\bar{P}_{\mathrm{I}, \mathrm{II}}= & P_{\mathrm{S}} E+\frac{P}{2 \pi}\left[\mp \frac{1}{2} \pi \pm 2 e \cos \omega+\left(\frac{3}{4} e^{2}+\frac{1}{8} e^{4}\right) \sin 2 \omega\right. \\
& \left.\mp\left(\frac{1}{3} e^{3}+\frac{1}{8} e^{5}\right) \cos 3 \omega-\frac{5}{32} e^{4} \sin 4 \omega \pm \frac{3}{40} e^{5} \cos 5 \omega\right],
\end{aligned}
$$

where $P_{\mathrm{s}}$ is the sidereal (or eclipsing) period of, for example, the first cycle, and $E$ the cycle-number. In the present case the orbital elements cease to remain constant. Nevertheless, as can be seen from Eqs. (8)-(14), their variation on the $P_{2}$ time scale is related to $P_{1} / P_{2} \ll 1$, which allows linearization of the problem; i.e., in such a case, Eq. (5) is formally valid in the same form, but $e, \omega$, and $P_{\mathrm{s}}$ are no longer constant. Then a further integration of Eq. (5) with respect to $v_{2}$ gives the analytical form of the perturbed $\mathrm{O}-\mathrm{C}$ on the $P_{2}$ time scale. To this, as a next step, we have to calculate the long-period and apse-node perturbations in $u$. Some of these arise simply from the similar perturbations of the orbital elements (or directly of the $e \cos \omega, e \sin \omega$ functions), while others (we refer to them as direct perturbations in u) come from the variations in the mean motion (for more details see Borkovits et al. 2007) ${ }^{2}$. (In other words this means that in such a case $P$ will no longer be constant.)

At this point, to avoid any confusion, we emphasize that during our calculations we use different sets of the angular orbital elements. As we are interested in a phenomenon that primarily depends on the relative positions of the orbiting celestial bodies with respect to the observer, the angular elements (i.e. $u, \omega, i$, $\Omega$ ) should be expressed in the "observational" frame of reference with the plane of the sky as the fundamental plane, and $u$, as well as $\omega$, is measured from the intersection of the binary's orbital plane with that plane, while $\Omega$ is measured along the plane of the sky from an arbitrary origin. On the other hand, the physical variations in the motion of the bodies depend on their positions relative to each other, and it is consequently more beneficial (and convenient) to express the equations of perturbations of the orbital elements in a different frame of reference (we refer to it as the dynamical frame), determined only by the relative positions of the bodies. The fundamental plane of this frame of reference is the invariable plane of the triple system, i.e. the plane perpendicular to the net angular momentum vector of the complete triple system. In this frame of reference, the longitude of the ascending node $(h)$ gives the arc between the sky and the corresponding intersection of the two orbital planes measured along the invariable plane, while the true longitude $(w)$ and the argument of periastron $(g)$ are measured from that ascending node, along the respected orbital plane. To avoid a further confusion,

2 The minus signs on the r.h.s. of Eqs. (10) and (11) of Borkovits et al. (2007) should be replaced by plus. 


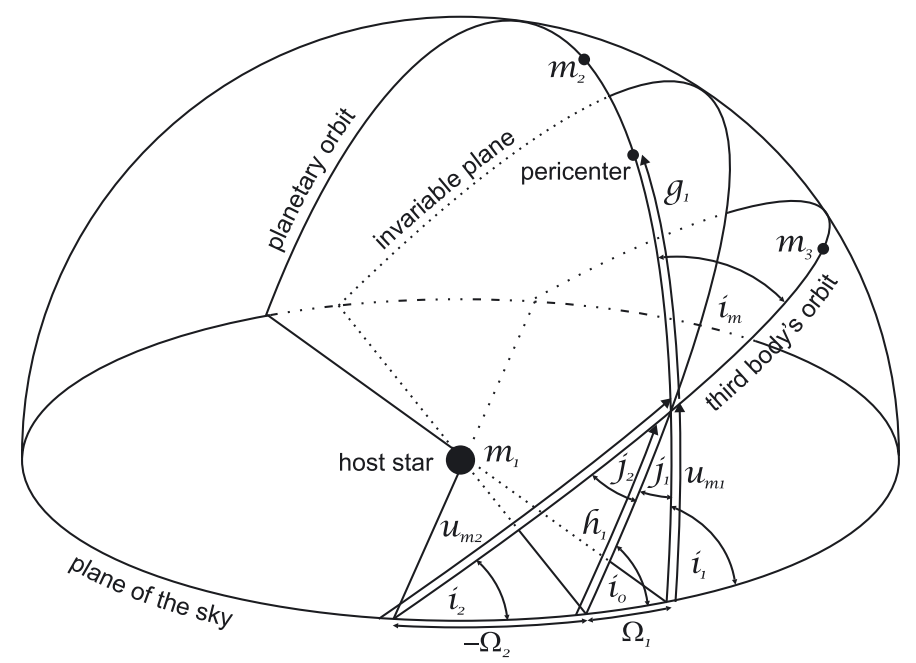

Fig. 1. The spatial configuration of the system.

the relative inclination of the orbits to the invariable plane is denoted by $j$. The meaning and the relation between the different elements can be seen in Fig. 1, and are also listed in Appendix A.

\subsection{Long-period perturbations}

From now on we refer to the orbital elements of the inner binary (i.e. the pair formed by either a host star and the inner planet, or two stellar mass objects) by subscript ${ }_{1}$, while those of the wider binary (i.e. the orbit of the third component around the centre of mass of the inner system) by subscript 2 . The differential perturbation equations of the orbital elements are listed in Borkovits et al. (2007) ${ }^{3}$. In order to get the long-period terms of the perturbation equations, the usual method involves averaging the equations for the short-period $\left(\approx P_{1}\right)$ variables, which is usually the mean anomaly $\left(l_{1}\right)$ or the true anomaly $\left(v_{1}\right)$ of the inner binary, but in our special case it is the true longitude $u_{1}$. This means that we get the variation in the orbital elements averaged over an eclipsing period. Furthermore, in the case of the averaged equations we change the independent variable from $u_{1}$ to (the averaged) $v_{2}$, by the use of

$\frac{\mathrm{d} u_{1}}{\mathrm{~d} v_{2}} \approx \frac{\rho_{2}^{2}}{c_{2}} \frac{c_{1}}{\rho_{1}^{2}}\left(1-\frac{\rho_{1}^{2}}{c_{1}} \dot{\Omega}_{1} \cos i_{1}\right)$,

from which, after averaging, we get

$$
\begin{aligned}
\frac{\mathrm{d} \overline{u_{1}}}{\mathrm{~d} \overline{v_{2}}} & \approx \frac{\mu_{1}^{1 / 2}}{a_{1}^{3 / 2}} \frac{\rho_{2}^{2}}{\sqrt{\mu_{2} a_{2}\left(1-e_{2}^{2}\right)}}-\frac{\mathrm{d} \overline{\Omega_{1}}}{\mathrm{~d} \overline{v_{2}}} \cos i_{1} \\
& \approx \frac{P_{2}}{P_{1}} \frac{\left(1-e_{2}^{2}\right)^{3 / 2}}{\left(1+e_{2} \cos v_{2}\right)^{2}}-\frac{\mathrm{d} \Omega_{1}}{\mathrm{~d} v_{2}} \cos i_{1} .
\end{aligned}
$$

In the following we omit the overlining. Furthermore, as one can see from Eq. (13) below, the second term on the r.h.s. of (7)

\footnotetext{
${ }^{3}$ In that paper, from practical considerations, we did not restrict ourselves to the most usual representation of the perturbation equations, i.e. the Lagrange equations with the perturbing function; rather, we used the somewhat more general form that expresses the perturbations with the three orthogonal components of the perturbing force. Nevertheless, as long as only conservative, three-body terms are considered, the two representations are perfectly equivalent. (In the denominator of Eqs. (14) and (15) for $e$ and $\omega$, a closing bracket is evidently missing, and furthermore, the equation for $\Omega$ should be divided by $e$ for the correct result.)
}

is of the order $P_{1} / P_{2}$, so can be neglected. For the long-period perturbations of the orbital elements of the close orbit we get$$
\frac{\mathrm{d} a_{1}}{\mathrm{~d} v_{2}}=0,
$$$$
\frac{\mathrm{d} e_{1}}{\mathrm{~d} v_{2}}=A_{\mathrm{L}}\left(1-e_{1}^{2}\right)^{1 / 2} e_{1}\left(1+e_{2} \cos v_{2}\right)
$$$$
\times\left[\left(1-I^{2}\right) \sin 2 g_{1}\right.
$$$$
-\frac{1}{2}(1+I)^{2} \sin \left(2 v_{2}+2 g_{2}-2 g_{1}\right)
$$$$
\left.+\frac{1}{2}(1-I)^{2} \sin \left(2 v_{2}+2 g_{2}+2 g_{1}\right)\right] \text {, }
$$$$
\frac{\mathrm{d} g_{1}}{\mathrm{~d} v_{2}}=A_{\mathrm{L}}\left(1-e_{1}^{2}\right)^{1 / 2}\left(1+e_{2} \cos v_{2}\right)
$$$$
\times\left\{\frac{3}{5}\left(I^{2}-\frac{1}{3}\right)\right.
$$$$
+\left(1-I^{2}\right)\left[\cos 2 g_{1}+\frac{3}{5} \cos \left(2 v_{2}+2 g_{2}\right)\right]
$$$$
+\frac{1}{2}(1+I)^{2} \cos \left(2 v_{2}+2 g_{2}-2 g_{1}\right)
$$$$
\left.+\frac{1}{2}(1-I)^{2} \cos \left(2 v_{2}+2 g_{2}+2 g_{1}\right)\right\}
$$$$
-\frac{\mathrm{d} h_{1}}{\mathrm{~d} v_{2}} \cos j_{1},
$$

$$
\begin{aligned}
\frac{\mathrm{d} h_{1}}{\mathrm{~d} v_{2}}= & -A_{\mathrm{L}}\left(1-e_{1}^{2}\right)^{-1 / 2} \frac{\sin i_{\mathrm{m}}}{\sin j_{1}}\left(1+e_{2} \cos v_{2}\right) \\
& \times\left[\frac{2}{5}\left(1+\frac{3}{2} e_{1}^{2}\right) I\right. \\
& -e_{1}^{2} I \cos 2 g_{1} \\
& -\frac{2}{5}\left(1+\frac{3}{2} e_{1}^{2}\right) I \cos \left(2 v_{2}+2 g_{2}\right) \\
& +\frac{1}{2} e_{1}^{2}(1+I) \cos \left(2 v_{2}+2 g_{2}-2 g_{1}\right) \\
& \left.-\frac{1}{2} e_{1}^{2}(1-I) \cos \left(2 v_{2}+2 g_{2}+2 g_{1}\right)\right],
\end{aligned}
$$$$
\frac{\mathrm{d} j_{1}}{\mathrm{~d} v_{2}}=A_{\mathrm{L}}\left(1-e_{1}^{2}\right)^{-1 / 2} \sin i_{\mathrm{m}}\left(1+e_{2} \cos v_{2}\right)
$$$$
\times\left[\frac{2}{5}\left(1+\frac{3}{2} e_{1}^{2}\right) \sin \left(2 v_{2}+2 g_{2}\right)\right.
$$$$
+e_{1}^{2} I \sin 2 g_{1}
$$$$
-\frac{1}{2} e_{1}^{2}(1+I) \sin \left(2 v_{2}+2 g_{2}-2 g_{1}\right)
$$$$
\left.+\frac{1}{2} e_{1}^{2}(1-I) \sin \left(2 v_{2}+2 g_{2}+2 g_{1}\right)\right] \text {, }
$$

$$
\begin{aligned}
\frac{\mathrm{d} \omega_{1}}{\mathrm{~d} v_{2}}= & A_{\mathrm{L}}\left(1-e_{1}^{2}\right)^{1 / 2}\left(1+e_{2} \cos v_{2}\right) \\
& \times\left\{\frac{3}{5}\left(I^{2}-\frac{1}{3}\right)\right. \\
& +\left(1-I^{2}\right)\left[\cos 2 g_{1}+\frac{3}{5} \cos \left(2 v_{2}+2 g_{2}\right)\right] \\
& +\frac{1}{2}(1+I)^{2} \cos \left(2 v_{2}+2 g_{2}-2 g_{1}\right) \\
& \left.+\frac{1}{2}(1-I)^{2} \cos \left(2 v_{2}+2 g_{2}+2 g_{1}\right)\right\}-\frac{\mathrm{d} \Omega_{1}}{\mathrm{~d} v_{2}} \cos i_{1}
\end{aligned}
$$


T. Borkovits et al.: Transit timing variations in eccentric hierarchical triple exoplanetary systems. I.

$$
\begin{aligned}
\frac{\mathrm{d} \Omega_{1}}{\mathrm{~d} v_{2}}= & A_{\mathrm{L}}\left(1-e_{1}^{2}\right)^{-1 / 2} \frac{\sin i_{\mathrm{m}}}{\sin i_{1}}\left(1+e_{2} \cos v_{2}\right) \\
& \times\left[\frac{2}{5}\left(1+\frac{3}{2} e_{1}^{2}\right) I \cos \left(\omega_{1}-g_{1}\right)\right. \\
& +\frac{1}{5}\left(1+\frac{3}{2} e_{1}^{2}\right)(1-I) \cos \left(2 v_{2}+2 g_{2}-\omega_{1}+g_{1}\right) \\
& -\frac{1}{5}\left(1+\frac{3}{2} e_{1}^{2}\right)(1+I) \cos \left(2 v_{2}+2 g_{2}+\omega_{1}-g_{1}\right) \\
& -e_{1}^{2} I \cos \left(\omega_{1}+g_{1}\right) \\
& +\frac{1}{2} e_{1}^{2}(1+I) \cos \left(2 v_{2}+2 g_{2}-\omega_{1}-g_{1}\right) \\
& \left.-\frac{1}{2} e_{1}^{2}(1-I) \cos \left(2 v_{2}+2 g_{2}+\omega_{1}+g_{1}\right)\right], \\
\frac{\mathrm{d} i_{1}}{\mathrm{~d} v_{2}}= & A_{\mathrm{L}}\left(1-e_{1}^{2}\right)^{-1 / 2} \sin i_{\mathrm{m}}\left(1+e_{2} \cos v_{2}\right) \\
& \times\left[-\frac{2}{5}\left(1+\frac{3}{2} e_{1}^{2}\right) I \sin \left(\omega_{1}-g_{1}\right)\right. \\
& +\frac{1}{5}\left(1+\frac{3}{2} e_{1}^{2}\right)(1-I) \sin \left(2 v_{2}+2 g_{2}-\omega_{1}+g_{1}\right) \\
& +\frac{1}{5}\left(1+\frac{3}{2} e_{1}^{2}\right)(1+I) \sin \left(2 v_{2}+2 g_{2}+\omega_{1}-g_{1}\right) \\
& +e^{2} I \sin \left(\omega_{1}+g_{1}\right) \\
& -\frac{1}{2} e_{1}^{2}(1+I) \sin \left(2 v_{2}+2 g_{2}-\omega_{1}-g_{1}\right) \\
& \left.\sin \left(2 v_{2}+2 g_{2}+\omega_{1}+g_{1}\right)\right] . \\
& \\
&
\end{aligned}
$$

Finally, the direct term is

$$
\begin{aligned}
\frac{\mathrm{d} \lambda_{1}}{\mathrm{~d} v_{2}}= & A_{\mathrm{L}}\left(1-e_{1}^{2}\right)^{1 / 2}\left(1+e_{2} \cos v_{2}\right) \\
& \times\left\{\frac{4}{5}\left(I^{2}-\frac{1}{3}\right) f_{1}\left(e_{1}\right)\right. \\
& +\frac{51}{20}\left(1-I^{2}\right) e_{1}^{2} f_{2}\left(e_{1}\right) \cos 2 g_{1} \\
& +\frac{4}{5}\left(1-I^{2}\right) f_{1}\left(e_{1}\right) \cos \left(2 v_{2}+2 g_{2}\right) \\
& +\frac{51}{40}(1+I)^{2} e_{1}^{2} f_{2}\left(e_{1}\right) \cos \left(2 v_{2}+2 g_{2}-2 g_{1}\right) \\
& \left.+\frac{51}{40}(1-I)^{2} e_{1}^{2} f_{2}\left(e_{1}\right) \cos \left(2 v_{2}+2 g_{2}+2 g_{1}\right)\right\},
\end{aligned}
$$

where

$A_{\mathrm{L}}=\frac{15}{8} \frac{m_{3}}{m_{123}} \frac{P_{1}}{P_{2}}\left(1-e_{2}^{2}\right)^{-3 / 2}$

and

$f_{1}(e)=1+\frac{25}{8} e^{2}+\frac{15}{8} e^{4}+\frac{95}{64} e^{6}$ $f_{2}(e)=1+\frac{31}{51} e^{2}+\frac{23}{48} e^{4}$.

Furthermore, $i_{\mathrm{m}}$ denotes the mutual inclination of the two orbital planes, while

$I=\cos i_{\mathrm{m}}$,

and $m_{123}$ stands for the total mass of the system. Formal integration of the first five of the equations above (i.e. those that refer to the orbital elements in the dynamical system, Eqs. (8)-(11)) reproduce the results of Söderhjelm (1982).

Strictly speaking, some of the equations above are only valid when both orbits are non-circular, and the orbital planes are inclined to each other and/or to the plane of the sky. For example, if the outer orbit is circular, neither $v_{2}$ nor $g_{2}$ has any meaning. Nevertheless, their sum i.e. $v_{2}+g_{2}$ is meaningful, as before. Similarly, although the derivative of $g_{1}$ has no meaning in the case of a circular inner orbit, the derivatives of $e_{1} \cos g_{1}, e_{1} \sin g_{1}$ (or $e_{1} \cos \omega_{1}, e_{1} \sin \omega_{1}$ ), i.e. the so-called Lagrangian elements can be calculated correctly. (Instead of the "pure" derivatives of $e_{1}$ and $g_{1}$ (or $\left.\omega_{1}\right)$ these occur directly in the O-C.) Similar redefenitions can be done in the case of coplanarity. For practical reasons and for the sake of clarity, we therefore retain the original formulations, even in such cases, when it is formally not valid.

As one can see, there are some terms on the r.h.s. of these equations that do not depend on $v_{2}$. Primarily, these terms give the so-called apse-node time-scale contribution to the variation in the orbital elements and the TTVs. Nevertheless, to get a correct result for the long-term behaviour of the orbital elements, these terms must nevertheless be retained in our long-term formulae. These terms were calculated for tidally distorted triplets in Borkovits et al. (2007). Such formulae (after omitting the tidal terms) are also valid for the present case in the low mutual inclination (i.e. approx. $I^{2}>\frac{3}{5}$ ) domain. (Formulae valid for arbitrary mutual inclinations will be presented in a subsequent paper.)

Carrying out the integrations, all the orbital elements on the r.h.s. of these equations with the exception of $v_{2}$ are considered as constants. This can be justified for two reasons. First, as one can see, the amplitudes of the long-period perturbative terms $\left(A_{\mathrm{L}}\right)$ remain small for $P_{2} \gg P_{1}$ (which is especially valid for the case where the host star is orbited by two planets, when $\left.m_{3} \ll m_{123}\right)^{4}$, and second, although the amplitude of the apsenode perturbative terms can reach unity, the period is usually so long $\left(\sim P_{2}^{2} / P_{1}\right.$, see e.g. Brown 1936) that its contribution can be safely ignored during one revolution of the outer object ${ }^{5}$. The

${ }_{4}$ This assumption is analogous to that of the classical low-order perturbation theory where the squares of the orbital element changes are neglected, as they should be proportional to $\left(m_{\text {perturber }} / m_{\text {star }}\right)^{2}$, but with the difference that here the small parameter is the ratio of the semi-major axes $a_{1} / a_{2}$ instead of the mass ratio. Consequently, this assumption remains valid even if the perturber is a sufficiently distant stellar-mass object.

5 This is not necessarily true in the presence of other perturbative effects. Nevertheless, in such cases one can assume that if there is a physical effect producing apse-node time scale perturbations having a period comparable to the period of the long-period perturbations arising from some other sources, then the amplitudes of these long-period perturbations are usually so small with respect to the amplitudes of the apsenode perturbations that their effect can be neglected. 
final result of such an analysis is an analytical form of the TTVs, and i.e. the long-period $\mathrm{O}-\mathrm{C}$ diagram:

$$
\begin{aligned}
\mathrm{O}-\mathrm{C}_{P_{2}}= & \frac{P_{1}}{2 \pi} A_{\mathrm{L}}\left(1-e_{1}^{2}\right)^{1 / 2}\left\{\left(\frac{4}{5}+\frac{5}{2} e_{1}^{2} \mp \frac{6}{5} e_{1} \sin \omega_{1}\right)\right. \\
& \times\left[\left(I^{2}-\frac{1}{3}\right) \mathcal{M}+\frac{1}{2}\left(1-I^{2}\right) \mathcal{S}\left(2 v_{2}+2 g_{2}\right)\right] \\
& +\left[\frac{51}{20} e_{1}^{2} \cos 2 g_{1} \mp 2 e_{1} \sin \left(\omega_{1}-2 g_{1}\right)\right] \\
& \times\left[\left(1-I^{2}\right) \mathcal{M}+\frac{1}{2}\left(1+I^{2}\right) \mathcal{S}\left(2 v_{2}+2 g_{2}\right)\right] \\
& -\left[\frac{51}{20} e_{1}^{2} \sin 2 g_{1} \mp 2 e_{1} \cos \left(\omega_{1}-2 g_{1}\right)\right] I C\left(2 v_{2}+2 g_{2}\right) \\
& +\cot i_{1} \sin i_{\mathrm{m}}\left\{-\frac{2}{5}\left(1 \mp 2 e_{1} \sin \omega_{1}\right) \cos u_{\mathrm{m} 1} I\right. \\
& \times\left[\mathcal{M}-\frac{1}{2} \mathcal{S}\left(2 v_{2}+2 g_{2}\right)\right] \\
& \left.\left.+\frac{1}{5}\left(1 \mp 2 e_{1} \sin \omega_{1}\right) \sin u_{\mathrm{m} 1} C\left(2 v_{2}+2 g_{2}\right)\right\}\right\} \\
& +\frac{m_{3}}{m_{123}} \frac{a_{2} \sin i_{2}}{c} \frac{\left(1-e_{2}^{2}\right) \sin \left(v_{2}+\omega_{2}\right)}{1+e_{2} \cos v_{2}} \\
& +O\left(e_{1}^{2}\right),
\end{aligned}
$$

$u_{\mathrm{m} 1}=\omega_{1}-g_{1}$

i.e., $u_{\mathrm{m} 1}$ is the angular distance of the intersection of the two orbits from the plane of the sky, or, in other words, the longitude of the (dynamical) ascending node of the inner orbit along the orbital plane, measured from the sky. Here we give the result up to the first order in the inner eccentricity, while a more extended result up to the sixth order in the inner eccentricity can be found in Appendix B, where we give also the perturbation equations directly for the $e_{1}^{m} \cos n \omega_{1}, e_{1}^{m} \sin n \omega_{1}$ expressions. The upper signs refer to the exoplanetary transits (primary minima), and the lower signs to the secondary occultations (secondary minima). Furthermore, we assumed the formally second-order $\frac{5}{2} e_{1}^{2}$, and $\frac{51}{20} e_{1}^{2}$ terms to be first order, as their values exceed $e_{1}$ for medium eccentricities. We also included the pure geometrical light-time contribution, shown in the last row. Here $c$ denotes the speed of light. The minus sign arises because this term reflects the motion of the inner pair around the common centre of mass, whose true longitude differs by $\pi$ from the one of the third component. In Eq. (21) the mean anomaly of the outer body $\left(l_{2}\right)$ appears because of (the constant part of) the difference between the anomalistic $P$ and sideral (eclipsing, or transiting) $P_{\text {s }}$ period included into the first term on the r.h.s. of Eq. (5).

where

$$
\begin{aligned}
\mathcal{M} & =\int 1+e_{2} \cos v_{2} \mathrm{~d} v_{2} \\
& =v_{2}-l_{2}+e_{2} \sin v_{2} \\
& =3 e_{2} \sin v_{2}-\frac{3}{4} e_{2}^{2} \sin 2 v_{2}+\frac{1}{3} e_{2}^{3} \sin 3 v_{2}+O\left(e_{2}^{4}\right) \\
& =3 e_{2}\left(1-\frac{3}{8} e_{2}^{2}\right) \sin l_{2}+\frac{9}{4} e_{2}^{2} \sin 2 l_{2}+\frac{53}{24} e_{2}^{3} \sin 3 l_{2}+O\left(e_{2}^{4}\right),
\end{aligned}
$$

and

$$
\begin{aligned}
\mathcal{S} & \left(2 v_{2}+x\right)=\sin \left(2 v_{2}+x\right)+e_{2} \sin \left(v_{2}+x\right)+\frac{1}{3} e_{2} \sin \left(3 v_{2}+x\right) \\
& =\left(1-4 e_{2}^{2}\right) \sin \left(2 l_{2}+x\right)+e_{2}\left[-\left(1-\frac{13}{8} e_{2}^{2}\right) \sin \left(l_{2}+x\right)\right. \\
& \left.+\frac{7}{3}\left(1-\frac{207}{56} e_{2}^{2}\right) \sin \left(3 l_{2}+x\right)\right]+e_{2}^{2}\left[-\frac{1}{4} \sin x+\frac{17}{4} \sin \left(4 l_{2}+x\right)\right] \\
& +e_{2}^{3}\left[\frac{1}{24} \sin \left(l_{2}-x\right)+\frac{169}{24} \sin \left(5 l_{2}+x\right)\right]+O\left(e_{2}^{4}\right) \\
C & \left(2 v_{2}+x\right)=\cos \left(2 v_{2}+x\right)+e_{2} \cos \left(v_{2}+x\right)+\frac{1}{3} e_{2} \cos \left(3 v_{2}+x\right) \\
& =\left(1-4 e_{2}^{2}\right) \cos \left(2 l_{2}+x\right) \\
& +e_{2}\left[-\left(1-\frac{13}{8} e_{2}^{2}\right) \cos \left(l_{2}+x\right)\right. \\
& \left.+\frac{7}{3}\left(1-\frac{207}{56} e_{2}^{2}\right) \cos \left(3 l_{2}+x\right)\right] \\
& +e_{2}^{2}\left[-\frac{1}{4} \cos x+\frac{17}{4} \cos \left(4 l_{2}+x\right)\right] \\
& +e_{2}^{3}\left[-\frac{1}{24} \cos \left(l_{2}-x\right)+\frac{169}{24} \cos \left(5 l_{2}+x\right)\right]+O\left(e_{2}^{4}\right)
\end{aligned}
$$

A53, page 6 of 25

\section{Discussion of the results}

As one can easily see, the result for a circular inner orbit (i.e. $e_{1}=0$ ) is identical with the formula (46) of Borkovits et al. (2003). Consequently, the discussion in Sect. 4 of that paper is also valid. Nevertheless, as one can also see in Figs. 2, 3, a significant inner eccentricity produces notably higher amplitudes and is consequently easier to detect. Furthermore, some other attributes of the TTVs also change drastically. For example, in contrast to the previously studied coplanar $(I= \pm 1)$, circular inner orbit $\left(e_{1}=0\right)$ case (Borkovits et al. 2003; Agol et al. 2005), the dynamical term does not disappear even if the outer orbit is circular $\left(e_{2}=0\right)$, as long as the inner orbit is eccentric. Another important feature for an eccentric inner orbit is that the amplitude, the phase, and the shape of the $\mathrm{O}-\mathrm{C}$ variations cease to depend simply on the physical (i.e. relative) positions of the celestial bodies, but also on the orientation of the orbit with respect to the observer. These last elements (i.e. $e_{1} \sin \omega_{1}, e_{1} \cos \omega_{1}$, and their combinations) can also be determined from $(a)$ radial velocity measurements, $(b)$ the shape of the transit light curves, and $(c)$ the time delay between the two different eclipsing events. (Naturally, the last one requires the detection of the secondary occultations or minima.) While the relative, i.e., physical angular parameters (i.e. periastron distances from the intersection of the two orbits, $g_{1}, g_{2}$, and mutual inclination $i_{\mathrm{m}}$ ) cannot be aquired from other, generally used methods (e.g. light-curve, or simple radial velocity curve analysis), these observational geometrical parameters could be determined from other sources of information, and then can be simply built into such a fitting algorithm, which was described in Borkovits et al. (2003), or could be included in procedures like those presented by Pál (2010).

In the following we omit terms multiplied by $\cot i_{1}$, because for the considered relatively longer period transiting systems $i_{1} \approx 90^{\circ}$ holds. In Borkovits et al. (2003) we only considered triple stellar systems, where the three masses were usually nearly equal, and the inner period was a few days. In such cases, the 
T. Borkovits et al.: Transit timing variations in eccentric hierarchical triple exoplanetary systems. I.
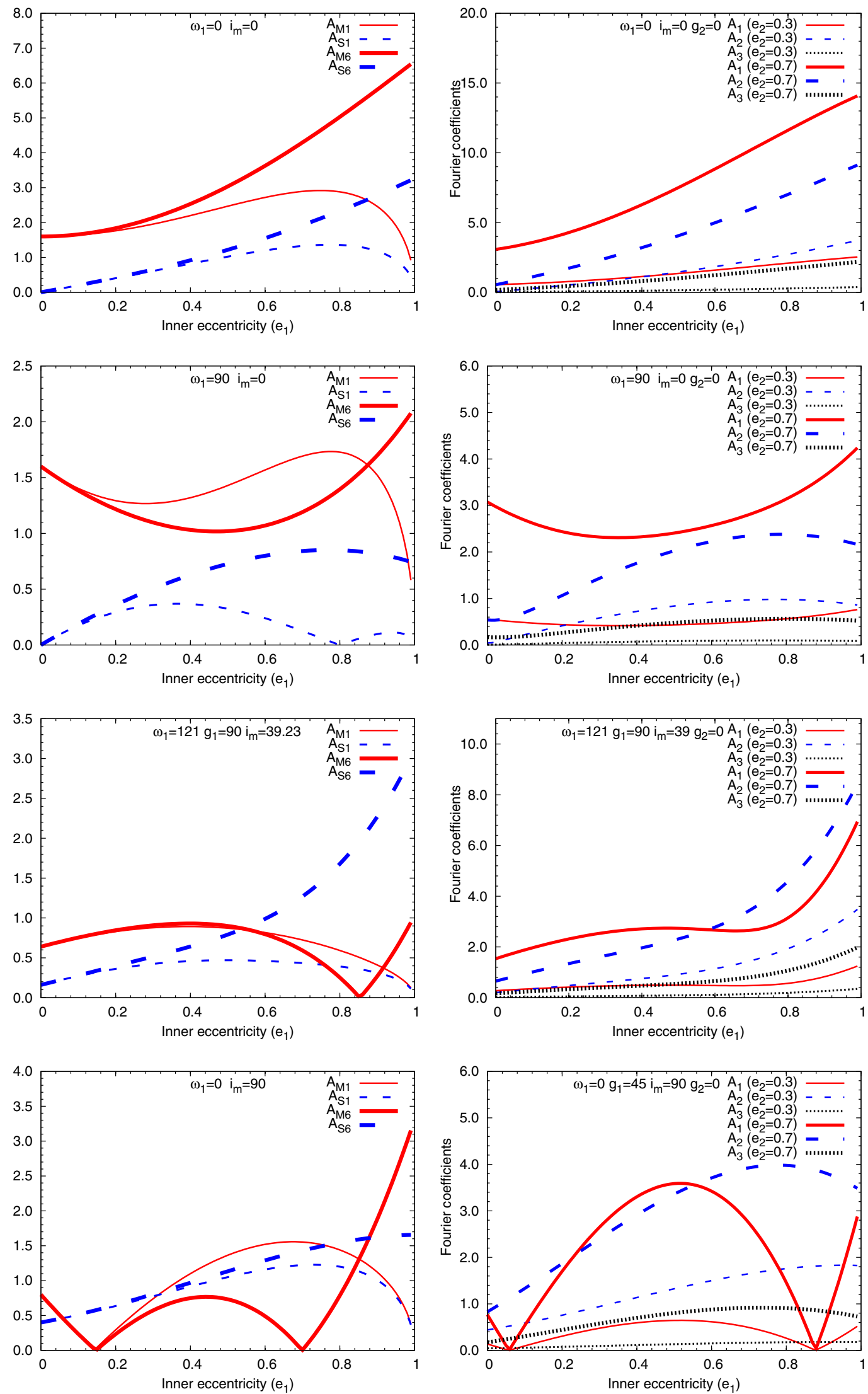

Fig. 2. Left panels: the dependence on the inner eccentricity $\left(e_{1}\right)$ of the amplitudes $A_{\mathcal{M}}, A_{\mathcal{S}}$ of the functions $\mathcal{M}, \mathcal{S}$ describing the long-period dynamical part of $\mathrm{O}-\mathrm{C}$ for specific values of other orbital elements. To compare the $\mathcal{M}$ and $\mathcal{S}$ terms, the former should be multiplied by $e_{2}$. The thin lines (indexed by " 1 ") refer to the first-order approximation, and the thick ones (index "6") to the sixth one. Right panels: the corresponding $A_{1,2,3}$ amplitudes of the trigonometric representation (Eq. (32)) of the $\mathrm{O}-\mathrm{C}$ for two different outer eccentricities $\left(e_{2}=0.3\right.$ and $\left.e_{2}=0.7\right)\left(g_{2}\right.$ was set to $\left.0^{\circ}\right)$. 

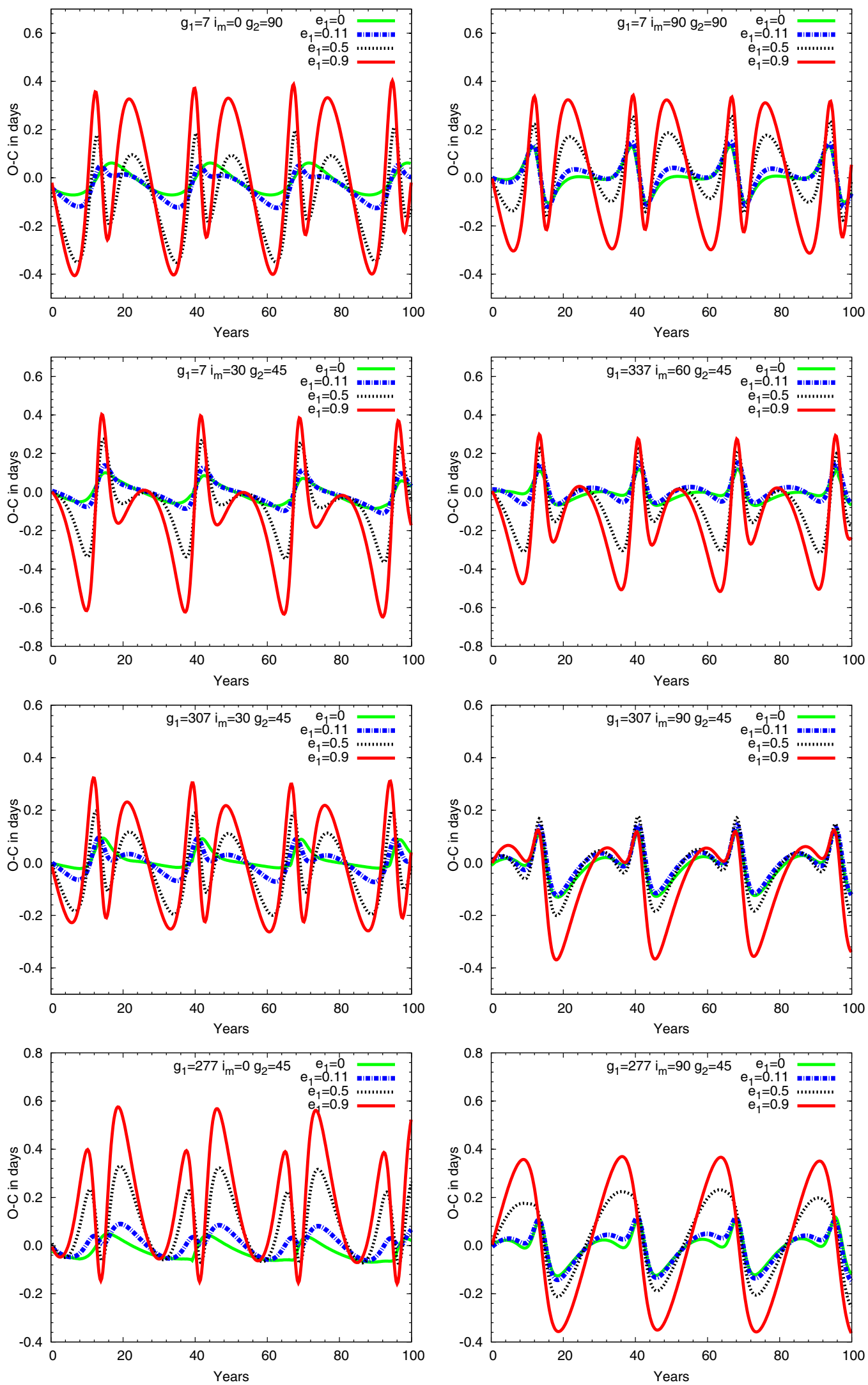

Fig. 3. Transit timing variatons caused by a hypothetical $P_{2}=10000$ day-period $m_{3}=1 M_{\odot}$ mass, moderately eccentric $e_{2}=0.3$ third companion for CoRoT-9b, at different initial orbital elements for four different initial inner eccentricities $\left(e_{1}=0,0.11,0.5,0.9\right)$. The various initial elements of each panel are as follows. Panel a): $\left.g_{1}=7^{\circ}, g_{2}=90^{\circ}, i_{\mathrm{m}}=0^{\circ} ; \mathbf{b}\right)$ : the same, but for $\left.\left.i_{\mathrm{m}}=90^{\circ} ; \mathbf{c}\right): g_{1}=7^{\circ}, g_{2}=45^{\circ}, i_{\mathrm{m}}=30^{\circ} ; \mathbf{d}\right): g_{1}=337^{\circ}$, $\left.\left.g_{2}=45^{\circ}, i_{\mathrm{m}}=60^{\circ} ; \mathbf{e}\right): g_{1}=307^{\circ}, g_{2}=45^{\circ}, i_{\mathrm{m}}=30^{\circ} ; \mathbf{f}\right):$ same as previous, but for $\left.\left.i_{\mathrm{m}}=90^{\circ} ; \mathbf{g}\right): g_{1}=277^{\circ}, g_{2}=45^{\circ}, i_{\mathrm{m}}=0^{\circ} ; \mathbf{h}\right): s a m e$ as previous, but for $i_{\mathrm{m}}=90^{\circ}$. (For better comparison the curves are corrected for the different average transit periods, and zero point shifts.) 
T. Borkovits et al.: Transit timing variations in eccentric hierarchical triple exoplanetary systems. I.

light-time term dominates. The amplitude of the light-time effect is simply

$$
\begin{aligned}
A_{\text {LITE }} & =\frac{m_{3}}{m_{123}} \frac{a_{2} \sin i_{2}}{c}\left(1-e_{2}^{2} \cos ^{2} \omega_{2}\right)^{1 / 2} \\
& =\frac{m_{3}}{m_{123}}\left(\frac{G m_{123}}{4 \pi^{2}}\right)^{1 / 3} \frac{\sin i_{2}}{c} P_{2}^{2 / 3}\left(1-e_{2}^{2} \cos ^{2} \omega_{2}\right)^{1 / 2} \\
& \approx 1.1 \times 10^{-4} \frac{m_{3}}{m_{123}^{2 / 3}} \sin i_{2} P_{2}^{2 / 3}\left(1-e_{2}^{2} \cos ^{2} \omega_{2}\right)^{1 / 2}
\end{aligned}
$$

where masses should be expressed in solar units, and $P_{2}$ in days.

The amplitude of the dynamically forced $\mathrm{O}-\mathrm{C}$ and, consequently, the detectability limit of such perturbations depends on almost all the dynamical, as well as geometrical, variables. We can therefore give only some limits on the detectability limit. Nevertheless, for an easier, and somewhat general study, we separate the physical and geometrical variables from each other and, furthermore, also separate the elements of the inner orbit from those of the outer perturber (with the exception of the mutual inclination). To do this, we introduce the following quantities, all of which depend on eccentricity $\left(e_{1}\right)$, the two types of periastron arguments $\left(g_{1}, \omega_{1}\right)$, and mutual inclination $\left(i_{\mathrm{m}}\right)$, via its cosine:

$$
\begin{aligned}
\alpha= & \left\{\frac{2}{5}+\frac{5}{4} e_{1}^{2}\left(1+\cos 2 g_{1}\right) \mp e_{1}\left[\frac{3}{5} \sin \omega_{1}+\sin \left(\omega_{1}-2 g_{1}\right)\right]\right. \\
& -I^{2}\left\{\frac{2}{5}+\frac{5}{4} e_{1}^{2}\left(1-\cos 2 g_{1}\right)\right. \\
& \left.\left.\mp e_{1}\left[\frac{3}{5} \sin \omega_{1}-\sin \left(\omega_{1}-2 g_{1}\right)\right]\right\}\right\}\left(1-e_{1}^{2}\right)^{1 / 2}, \\
\beta= & -\left(1-e_{1}^{2}\right)^{1 / 2} 2 I\left[\frac{5}{4} e_{1}^{2} \sin 2 g_{1} \mp e_{1} \cos \left(\omega_{1}-2 g_{1}\right)\right] \\
\gamma= & \left\{-\frac{4}{15}+\frac{5}{2} e_{1}^{2}\left(\cos 2 g_{1}-\frac{1}{3}\right) \mp 2 e_{1}\left[\sin \left(\omega_{1}-2 g_{1}\right)-\frac{1}{5} \sin \omega_{1}\right]\right. \\
& +I^{2}\left\{\frac{4}{5}+\frac{5}{2} e_{1}^{2}\left(1-\cos 2 g_{1}\right)\right. \\
& \left.\left.\mp 2 e_{1}\left[\frac{3}{5} \sin \omega_{1}-\sin \left(\omega_{1}-2 g_{1}\right)\right]\right\}\right\}\left(1-e_{1}^{2}\right)^{1 / 2} .
\end{aligned}
$$

Then

$\mathrm{O}-\mathrm{C}_{\mathrm{dyn}} \approx \frac{P_{1}}{2 \pi} A_{\mathrm{L}}\left[\gamma \mathcal{M}+\sqrt{\alpha^{2}+\beta^{2}} \mathcal{S}\left(2 v_{2}+\phi\right)\right]$

where

$\phi=2 g_{2}+\arctan \frac{\beta}{\alpha}$

or, in trigonometric form,

$\mathrm{O}-\mathrm{C}_{\mathrm{dyn}} \approx \frac{P_{1}}{2 \pi} A_{\mathrm{L}}^{*} \sum_{n} A_{n} \sin \left(n v_{2}+\phi_{n}\right)$ where (up to third order in $e_{2}$ )

$$
\begin{aligned}
& A_{1}=\left(1-e_{2}^{2}\right)^{-3 / 2} e_{2} \sqrt{A_{\mathcal{S}}^{2}+A_{\mathcal{M}}^{2}+2 A_{\mathcal{S}} A_{\mathcal{M}} \cos \phi} \\
& A_{2}=\left(1-e_{2}^{2}\right)^{-3 / 2} \sqrt{A_{\mathcal{S}}^{2}+\frac{1}{16} e_{2}^{4} A_{\mathcal{M}}^{2}+\frac{1}{2} e_{2}^{2} A_{\mathcal{S}} A_{\mathcal{M}} \cos \phi} \\
& A_{3}=\left(1-e_{2}^{2}\right)^{-3 / 2} \frac{1}{3} e_{2} \sqrt{A_{\mathcal{S}}^{2}+\frac{1}{9} e_{2}^{4} A_{\mathcal{M}}^{2}+\frac{2}{3} e_{2}^{2} A_{\mathcal{S}} A_{\mathcal{M}} \cos \phi} \\
& \phi_{1}=\arctan \frac{\sin \phi}{\cos \phi+\frac{A_{\mathcal{M}}}{A_{\mathcal{S}}}} \\
& \phi_{2}=\arctan \frac{\sin \phi}{\cos \phi+\frac{1}{4} e_{2}^{2} \frac{A_{\mathcal{M}}}{A_{\mathcal{S}}}} \\
& \phi_{3}=\arctan \frac{\sin \phi}{\cos \phi+\frac{1}{9} e_{2}^{2} \frac{A_{\mathcal{M}}}{A_{\mathcal{S}}}}
\end{aligned}
$$

and

$A_{\mathcal{S}}=\sqrt{\alpha^{2}+\beta^{2}}$

$A_{\mathcal{M}}=3 \gamma$,

and, furthermore,

$A_{\mathrm{L}}^{*}=A_{\mathrm{L}}\left(1-e_{2}^{2}\right)^{3 / 2}$.

For a circular outer orbit $\left(e_{2}=0\right)$

$$
A_{1}=A_{3}=0 \text {, }
$$$$
A_{2}=A_{\mathcal{S}} \text {. }
$$

As one can see, both sets of amplitudes, i.e. $A_{\mathcal{M}, \mathcal{S}}$ and $A_{1,2,3}$ are independent of the real physical parameters of the current exoplanetary system. Furthermore, the dependence on the elements of the outer orbit (i.e. $e_{2}, g_{2}$ ) appears only in the $A_{1,2,3}$ Fourier amplitudes. The masses (or more accurately mass ratios) and the periods and period ratios (and indirectly the physical size of the system) only occur as a scaling parameter. In this way, the following general statements are valid for all hierarchical triple systems (as long as the initial model assumptions are valid). To get the real, i.e. physical values for the $\mathrm{O}-\mathrm{C}$ amplitudes in a given system, one must multiply the general systemindependent, dimensionless amplitudes by the system specific number $\frac{15}{16 \pi} \frac{P_{1}^{2}}{P_{2}} \frac{m_{3}}{m_{1}+m_{2}+m_{3}}$.

Considering first the (nearly) coplanar case, i.e. when $I \approx$ \pm 1 , then the (half-)amplitude of the two sinusoidals becomes

$$
\begin{aligned}
e_{2} A_{\mathcal{M} 0} & \approx \frac{8}{5}\left(1-e_{1}^{2}\right)^{1 / 2}\left(1+\frac{25}{8} e_{1}^{2} \mp \frac{3}{2} e_{1} \sin \omega_{1}\right) e_{2}, \\
A_{\mathcal{S} 0} & \approx 2\left(1-e_{1}^{2}\right)^{1 / 2} e_{1} \sqrt{1 \mp \frac{5}{2} e_{1} \sin \omega_{1}+\frac{25}{16} e_{1}^{2}} .
\end{aligned}
$$

This illustrates the above-mentioned fundamental differences to the previously-studied coplanar, $e_{1}=0$ case (Borkovits et al. 2003; Agol et al. 2005), as according to our new result for eccentric inner orbits, the dynamical term does not disappear even if the outer orbit is circular $\left(e_{2}=0\right)$. Furthermore, the amplitudes strongly depend on the orientation of the orbital axis with respect to the observer. When the apsidal line coincides (more or less) with the line of sight (i.e. $\omega_{1}= \pm 90^{\circ}$ ), there can be very significant differences both in the shape and amplitude of the primary (transit) and secondary (occultation) $\mathrm{O}-\mathrm{C}$ curves. However, when the apsidal line lies nearly in the sky, then 
these differences disappear. Another interesting feature of the $\omega_{1}= \pm 90^{\circ}$ configuration is that for eclipse events that occur around apastron, there is a full square under the square-root sign in the $\mathcal{S}$-term, with the root of $e_{1}=0.8$, which means that this term would disappear in this situation. Nevertheless, for such high eccentricity, the first order approximation is far from being valid, as is illustrated in Fig. 2.

For highly inclined $(I \approx 0)$ orbits the (half-)amplitudes are as follows:

$$
\begin{aligned}
e_{2} A_{\mathcal{M} 90} \approx & \frac{4}{5}\left(1-e_{1}^{2}\right)^{1 / 2}\left\{-1-\frac{25}{8} e_{1}^{2}\left(1-3 \cos 2 g_{1}\right)\right. \\
& \left. \pm \frac{3}{2} e_{1}\left[\sin \omega_{1}-5 \sin \left(\omega_{1}-2 g_{1}\right)\right]\right\} e_{2}, \\
A_{\mathcal{S} 90} \approx & \frac{2}{5}\left(1-e_{1}^{2}\right)^{1 / 2}\left\{1+\frac{25}{8} e_{1}^{2}\left(1+\cos 2 g_{1}\right)\right. \\
& \left.\mp \frac{3}{2} e_{1}\left[\sin \omega_{1}+\frac{5}{3} \sin \left(\omega_{1}-2 g_{1}\right)\right]\right\},
\end{aligned}
$$

and the phase of the $\mathcal{S}$-term is simply

$\phi=2 g_{2}$.

In this case another parameter, namely, the periastron distance of the inner planet from the intersection of the two orbital planes $\left(g_{1}\right)$ also plays an important role.

Finally we also mention a very specific case, the maximum eccentricity phase of the Kozai mechanism driven $e$-cycles. During this phase, $\cos 2 g_{1}$ takes one definite value, namely $\cos 2 g_{1}=-1$. Furthermore, the mutual inclination of the two orbits here reaches its minimum. The actual value depends on both the maximum mutual inclination $i_{\mathrm{m}}$ or, more strictly, on $j_{1}$ and the minimal inner eccentricity $e_{1}$. Nevertheless, in the case of an initially almost circular inner orbit, the minimum mutual inclination is almost independent of its maximum value and takes $j_{1}\left(\approx i_{\mathrm{m}}\right) \approx 39.23$ (or its retrograde counterpart, $j_{1}\left(\approx i_{\mathrm{m}}\right) \approx$ 140.77 ), i.e. $I^{2}=3 / 5$. For this scenario

$$
\begin{aligned}
e_{2} A_{\mathcal{M} \text { Kozai } 90} & \approx \frac{16}{25}\left(1-e_{1}^{2}\right)^{1 / 2}\left(1-\frac{25}{16} e_{1}^{2} \pm \frac{9}{4} e_{1} \sin \omega_{1}\right) e_{2}, \\
\alpha_{\text {Kozai }, 90} & =\left(1-e_{1}^{2}\right)^{1 / 2}\left(\frac{4}{25}-\frac{5}{3} e_{1}^{2} \pm \frac{34}{25} e_{1} \sin \omega_{1}\right), \\
\beta_{\text {Kozai,90 }} & =\mp\left(1-e_{1}^{2}\right)^{1 / 2} \frac{2}{5} \sqrt{15} e_{1} \cos \omega_{1} .
\end{aligned}
$$

In order to get a better overview of the parameter dependence of the formulae above, we investigate the $A_{\mathcal{M}, \mathcal{S}}$, as well as the $A_{1,2,3}$, amplitudes graphically. Due to the complex dependence of these amplitudes on many parameters, it is difficult to give general statements. Therefore we investigate only the dependence of the amplitudes on the inner eccentricity, while the effects of other parameters are considered in Sect. 4 for specific systems, where some of the parameters can be fixed.

Figure 2 shows the inner eccentricity $\left(e_{1}\right)$ dependence of the amplitudes for some specific values of the other parameters. In general, one sees that the $e_{1}$ increase with amplitude, but this growth usually remains within one order of magnitude. Although the shapes of the individual $\mathrm{O}-\mathrm{C}$ curves may differ significantly, the net amplitudes vary over a narrow range. The graphs also suggest another, perhaps suprising, fact that the amplitude of $\mathrm{O}-\mathrm{C}_{\mathrm{P} 2 \text { dyn }}$ only weakly depends on the mutual inclination $\left(i_{\mathrm{m}}\right)$. This is especially valid for medium inner eccentricities, since in this case all $A_{\mathcal{M}, \mathcal{S}}$ amplitudes have similar values, at least for the cases shown in Fig. 2. Moreover, the numerically generated sample $\mathrm{O}-\mathrm{C}$ curves of Fig. 3 also suggest this conclusion. Nevertheless, there are several exceptions. There are particular configurations where one of the two amplitudes, or even both of them (and consequently, the corresponding terms), vanish. Such a situation is discussed in Sect. 4.1. We return to this question in detail in all of Sect. 4, where the dependence of the amplitudes on other parameters will also be studied for the systems of CoRoT-9b and HD 80606b.

Returning now to the LITE amplitude and comparing it with the dynamical case, we see that an increment of $P_{2}$ (keeping $P_{1}$ constant) results in an increment of $A_{\mathrm{LITE}} / A_{\mathrm{P} 2 \text {-dyn }}$ by $P_{2}^{5 / 3}$. Consequently, for more distant systems, the pure geometrical effect tends to exceed the dynamical one, as is the case in all but one ( $\lambda$ Tau, see e.g. Söderhjelm 1975) of the known hierarchical eclipsing triple stellar systems. Nevertheless, as we illustrate in this paper (see Fig. 5), we have a good chance of finding the opposite situation in some of the recently discovered transiting exoplanetary systems. We can make the following crude estimation. Consider a system with a solar-like host star, and two approximately Jupiter-mass companions) $\left(m_{123} \approx m_{1}=1 M_{\odot}\right.$, $\left.m_{2}=m_{3}=10^{-3} M_{\odot}\right)$, choosing $A=10^{-3}$ day for the case of a certain detection, then the LITE term for the most ideal case gives $P_{2} \geq 10^{6} \mathrm{~d} \approx 2700 \mathrm{y}$. Alternatively, after setting $m_{3}=10^{-2} M_{\odot}$ and allowing $A=10^{-4}$ for detection limit, the result is $P_{2} \geq 10^{3} \mathrm{~d} \approx 2.7 \mathrm{y}$. Similarly, for two Jupiter-mass planet, in the coplanar case, for small $e_{1}$, the $A=10^{-3}$ day limit gives the

$P_{2} \leq 2 \times 10^{3} m_{3} e_{2}\left(1-e_{2}^{2}\right)^{-3 / 2} P_{1}^{2}$

condition for the detectability of a third companion by its longterm dynamical perturbations. For the perpendicular case the same limit is

$$
P_{2} \leq \frac{1}{2} \times 10^{3} m_{3}\left(1-e_{2}^{2}\right)^{-3 / 2}\left(1+\frac{2}{3} e_{2}\right) P_{1}^{2} .
$$

We note that for $m_{3} \ll m_{1}$ the above equations are linear for $m_{3}$, so it is very easy to give the limiting period $P_{2}$ in the function of $m_{3}$. Nevertheless, we emphasize again that there are so many terms with different periodicities and phases that these equations give only a very crude first estimation. For $P_{1}=1,10,100$ days, (at zero outer eccentricity) gives $P_{2} \leq 0.5,50,5000$ days, respectively,

These results refer to the total amplitude of the O-C curve, i.e. the variation in the transit times during a complete revolution of the distant companion, which can take as long as several years or decades. Naturally, the perturbations in the transit times could be observed within a much shorter period, from the variation in the interval between consecutive minima. This estimation can be calculated, e.g., from Eq. (30) or even directly from Eq. (15). According to the meaning of the $\mathrm{O}-\mathrm{C}$ curve, the transiting or eclipsing period $(\bar{P})$ between two consecutive (let us assume the $n$th and $(n+1)$ th $)$ minima is

$$
\begin{aligned}
\bar{P}_{n}= & t_{n+1}-t_{n}=(\mathrm{O}-\mathrm{C})\left(t_{n+1}\right)-(\mathrm{O}-\mathrm{C})\left(t_{n}\right) \\
\approx & P_{\mathrm{S}}+\frac{P_{1}}{2 \pi} A_{\mathrm{L}}\left(1-e_{1}^{2}\right)^{1 / 2} \Delta v_{2 n}\left(1+e_{2} \cos v_{2 n}\right) \\
& \times\left[\gamma+2 \sqrt{\alpha^{2}+\beta^{2}} \cos \left(2 v_{2 n}+\phi\right)\right]
\end{aligned}
$$


T. Borkovits et al.: Transit timing variations in eccentric hierarchical triple exoplanetary systems. I.

where up to third order in $e_{2}$

$$
\begin{aligned}
\Delta v_{2} & \approx \Delta l_{2}\left(1+2 e_{2}^{2}+2 e_{2} \cos v_{2}+\frac{1}{2} e_{2}^{2} \cos 2 v_{2}+3 e_{2}^{3} \cos 3 v_{2}\right) \\
& \approx 2 \pi \frac{\bar{P}}{P_{2}}\left(1+\delta v_{2}\right) \\
& \approx 2 \pi \frac{P_{1}}{P_{2}}\left(1+\delta v_{2}\right) ;
\end{aligned}
$$

i.e., we approximated the variation of the mean anomaly of the outer companion $\left(l_{2}\right)$ by the constant value of

$\Delta l_{2} \approx 2 \pi \frac{P_{1}}{P_{2}}$.

(Here, and in the following text we neglect the pure geometrical LITE contribution.) Then the variation in the length of the consecutive transiting periods becomes

$$
\begin{aligned}
\Delta \bar{P} \approx & -\frac{15}{4} \pi \frac{m_{3}}{m_{123}} \frac{P_{1}^{4}}{P_{2}^{3}} \frac{\left(1-e_{1}^{2}\right)^{1 / 2}}{\left(1-e_{2}^{2}\right)^{3 / 2}} \\
& \times\left\{\gamma\left[3 e_{2}\left(1+\frac{9}{2} e_{2}^{2}\right) \sin v_{2}-6 e_{2}^{2} \sin 2 v_{2}-\frac{9}{2} e_{2}^{3} \sin 3 v_{2}\right]\right. \\
& +2 \sqrt{\alpha^{2}+\beta^{2}}\left[2\left(1+8 e_{2}^{2}\right) \sin \left(2 v_{2}+\phi\right)\right. \\
& +\frac{7}{2} e_{2}\left(1+\frac{9}{2} e_{2}^{2}\right) \sin \left(v_{2}+\phi\right) \\
& +\frac{13}{2} e_{2}\left(1+\frac{9}{2} e_{2}^{2}\right) \sin \left(3 v_{2}+\phi\right) \\
& \left.\left.+\frac{1}{4} e_{2}^{3} \sin (-v+\phi)+\frac{19}{4} e_{2}^{3} \sin \left(5 v_{2}+\phi\right)\right]\right\}
\end{aligned}
$$

Comparing (the amplitude of) this result with Eqs. $(1,2)$ of Holman \& Murray (2005), one can see that the power of the period ratio differs. (Furthermore, in the original paper $\pi$ stands at the same place, i.e. in the numerator as above but later, in Holman 2010, this was declared as an error, and it was put into the denominator.) There is a principal difference in the background. Holman \& Murray (2005) state that they "estimate the variation in transit intervals between successive transits", but what they really calculate is the departure of a transiting period (i.e. the interval between two successive transits) from a constant mean value. This quantity was estimated in our Eq. (53). In other words, the Lagrangian perturbation equations gives the instantaneous period of the perturbed system, so its integration gives the time left between two consecutive minima. The first derivative of the perturbation equation gives the instantaneous period variation, and so the variation in the length of two consecutive transiting period can be deduced by integrating the latter. As a consequence, the best possibility for detecting the TTV, hence for the presence of some perturber, occurs when the absolute value of the second derivative of the $\mathrm{O}-\mathrm{C}$ diagram, or

\begin{tabular}{|c|c|c|c|c|c|c|}
\hline System & $m_{1}$ & $m_{2}$ & $P_{1}$ & $e_{1}$ & $\omega_{1}$ & $i_{1}$ \\
\hline CoRoT $-9 b$ & $0.99 M_{\odot}$ & $0.0008 M_{\odot}$ & 95.2738 & 0.11 & $217^{\circ}$ & 89.99 \\
\hline HD 80606b & $0.97 M_{\odot}$ & $0.0038 M_{\odot}$ & 111.4357 & 0.93 & $121^{\circ}$ & 89.32 \\
\hline
\end{tabular}
practically Eq. (56) (the period variation during a revolution) is maximum. We illustrate this statement in the next section for specific systems.

\section{Case studies}

\subsection{CoRoT-9b}

CoRoT-9b is a transiting giant planet that revolves around its host star approximately at the distance of Mercury
Table 1. The initial parameters of the transiting planetary subsytems.

Notes. The parameters are taken from Deeg et al. (2010) and Pont et al. (2009).

(Deeg et al. 2010). Consequently, tidal forces (including the possible rotational oblateness) can safely be neglected in this system. Furthermore, thanks to the relatively large absolute separation of the planet from its star, we can expect a large amplitude signal from the perturbations of a hypothetical, distant (but not too distant) additional companion. To illustrate this possibility, and, furthermore, to check our formulae, we both calculated and plotted the amplitudes with the measured parameters of this specific system, and carried out short-term numeric integrations for comparison. The physical parameters and some of the orbital elements of CoRoT-9b were taken from Deeg et al. (2010). These data are listed in Table 1 . (We added $180^{\circ}$ to the $\omega_{1}$ published in that paper, as the spectroscopic $\omega_{1}$ refers to the orbit of the host star around the common centre of mass of the star-planet double system, so it differs by $180^{\circ}$ from the observational argument of periastron of the relative orbit of the transiting planet around its host star.) In the case of the numerical integrations, the inner planets were started from periastron and the outer one from its apastron.

Fixing the observationally aquired data, $A_{\mathcal{M}, \mathcal{S}}$ only depend on two parameters, namely $g_{1}$ and $i_{\mathrm{m}}$. In the left panels of Fig. 4, we plotted the $i_{\mathrm{m}}$ versus $A_{\mathcal{M}, \mathcal{S}}$ graphs for $g_{1}=69^{\circ}$ and $g_{1}=158^{\circ}$. We find that the amplitudes reach their extrema around these periastron arguments; i.e. for other $g_{1}$ values, results occur within the areas limited by these lines ${ }^{6}$. In the middle and right panels, the effect of the two other free parameters, i.e. outer eccentricity $\left(e_{2}\right)$, and dynamical (relative) argument of periastron $\left(g_{2}\right)$ were also considered. The middle panels show $A_{1,2}$ for $e_{2}=0.3$, the right panels for $e_{2}=0.7$, for both $g_{2}=0^{\circ}$ (upper panels), and $g_{2}=90^{\circ}$ (bottom panels). ( $A_{\mathcal{S}}$, plotted in the left panel is identical to $A_{2}$ for $e_{2}=0$, in which case $A_{1,3}=0$.)

These figures clearly show again that the amplitudes, and consequently, the actual full amplitude of the dynamical $\mathrm{O}-\mathrm{C}$ curve remains within one order of magnitude over a wide range of orbital parameters. This once more verifies the very crude estimations given by Eqs. (51), (52). As a consequence, for a given system we can very easily estimate the expected amplitude of the $\mathrm{O}-\mathrm{C}$ variations induced by an additional companion. For a planet-mass third body, the

$A_{\text {dyn }} \approx \frac{1}{2 \pi} \frac{m_{3}}{P_{2}} \frac{P_{1}^{2}}{m_{\text {host-star }}}$

gives a likely estimation at least in magnitude. For example, for CoRoT-9b,

$$
\begin{aligned}
\mathcal{A}=\frac{1}{2 \pi} \frac{P_{1}^{2}}{m_{\text {host-star }}} & \approx 1459 d^{2} M_{\odot}^{-1} \\
& \approx 1.39 d^{2} M_{\mathrm{J}}^{-1}
\end{aligned}
$$

i.e., a Jupiter-mass additional planet could produce 0.001 halfamplitude already from the distance of the Mars. (Of course, if

\footnotetext{
${ }^{6}$ Strictly speaking this argument is true only if negative values are also allowed for the coefficients.
} 

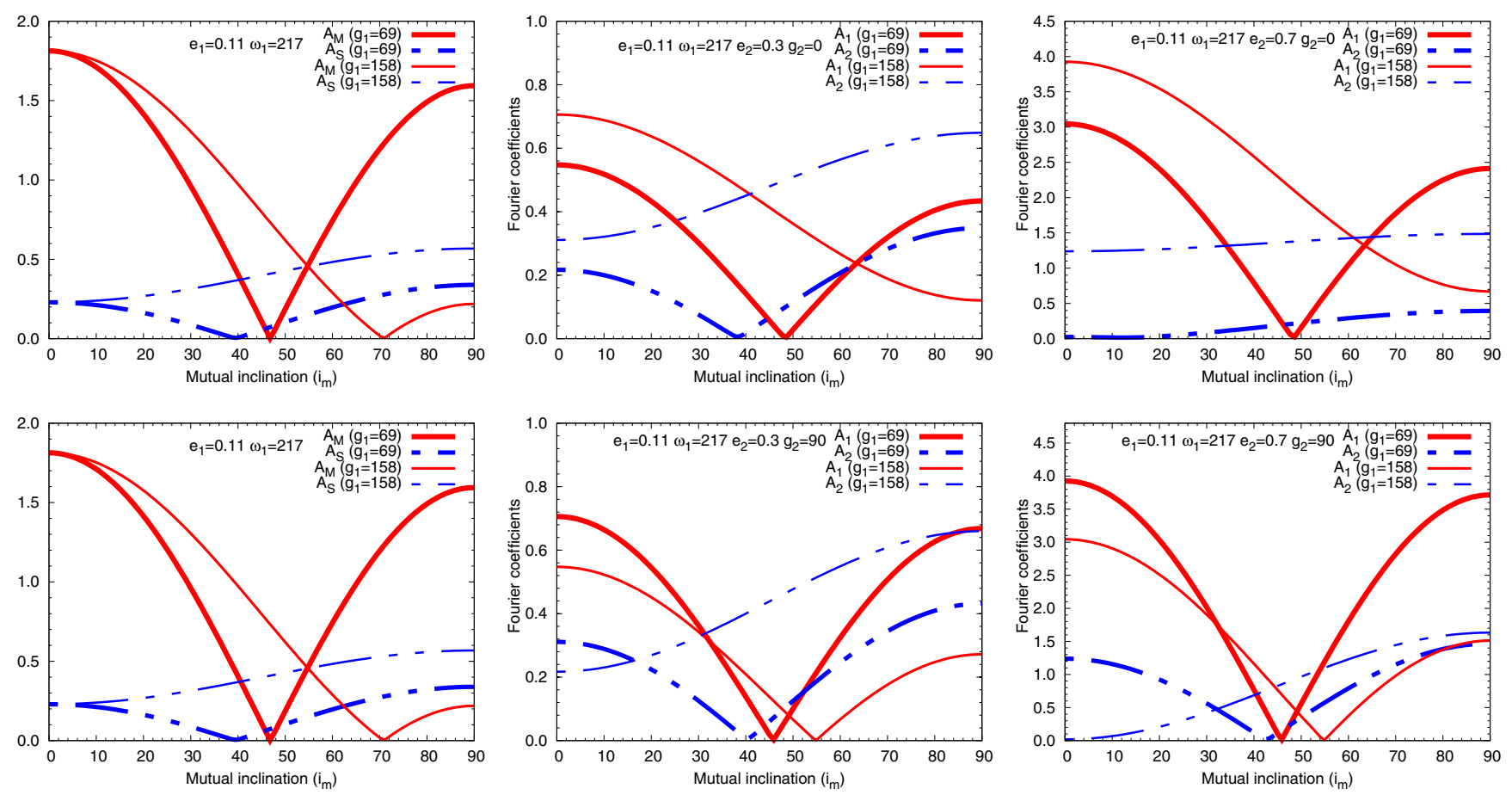

Fig. 4. Left panels: the dependence on the mutual inclination $\left(i_{\mathrm{m}}\right)$ of the amplitudes $A_{\mathcal{M}}, A_{\mathcal{S}}$ for CoRoT-9b, for $g_{1}=69^{\circ}$ and $g_{1}=158^{\circ}$. For other $g_{1}$ values the corresponding curves run within the area limited by these lines. (The two left panels are identical.) Middle and right panels: the corresponding $A_{1,2}$ amplitudes of the trigonometric representation (Eq. (32)) of the $\mathrm{O}-\mathrm{C}$ for two different outer eccentricities $e_{2}=0.3$ (middle) and $e_{2}=0.7$ (right); and outer dynamical (relative) argument of pericentrum arguments $g_{2}=0^{\circ}($ up $)$ and $g_{2}=90^{\circ}($ down). For the sake of clarity, we did not plot the small $A_{3}$ coefficients. (For $e_{2}=0.0$ the $A_{\mathcal{S}}$ amplitudes of the two identical left panels are equal to $A_{2}$, while $A_{1}=A_{3}=0$.)

$e_{1}$ and $\omega_{1}$ are known, a more precise estimation can be provided easily using the formulae of the present paper.)

Nevertheless, while the net amplitudes usually vary in a narrow range, the dominances of the two main terms (with period $P_{2}$ and $\left.\frac{1}{2} P_{2}\right)$ can alternate and, although it is not shown, their relative phase can also vary, so the shape of the actual curves may show great diversity, as is illustrated in Figs. 3, 5. Furthermore, for specific values of the parameters, one or other of the amplitudes might disappear. At CoRoT-9b particularly interesting is the $g_{1}=69^{\circ} i_{\mathrm{m}} \sim 45^{\circ}$ configuration since in this case both $A_{\mathcal{M}}$ and $A_{\mathcal{S}}$ disappear very close to each other. This means that the dynamical O-C almost disappears for specific $e_{2}, g_{2}$ values. This possibility warns us that from the absence of TTV in a given system one cannot automatically exclude the presence of an additional planet (which should be observed according to its parameters).

This can be seen clearly in Fig. 5, where we plotted the corresponding $\mathrm{O}-\mathrm{C}$ curves for coplanar, perpendicular, and the above-mentioned interesting $i_{\mathrm{m}}=40^{\circ}$ and $46^{\circ}$ configurations. In this figure we plotted $\mathrm{O}-\mathrm{C}$ curves obtained from both numerical integrations of the three-body motions and analytical calculations with our sixth-order formula. (According to Fig. 2, for the small inner eccentricity of CoRoT-9b, $e_{1}=0.11$, the firstorder approximation would have given practically the same results.) Comparing the analytical and the numerical curves, the best similarity can be seen in the perpendicular case (last row). In the coplanar case some minor discrepancies can be observed both in the shape and amplitude, while the discrepancies are expressed more in the particular $i_{\mathrm{m}}=46^{\circ}$ case, where for high outer eccentricity (right panel in the third row) our solution fails. (Nevertheless, the total amplitude of the analytical curve is similar to the numerical one in this case, too.) Although a thorough analysis of the sources of the discrepancies is beyond the scope of this paper, we suppose that in these situations the discrepancies come from the higher order perturbative terms. As was shown by Söderhjelm (1982) and Ford et al. (2000, among others), the higher order contributions are the most significant for $e_{1} \sim 0, i_{\mathrm{m}}=(0,1) \times 180^{\circ}$. Although these authors only considered the secular, or apse-node term perturbations, the same might be the case for the long-period ones. This might explain the better correspondence in the perpendicular configuration than in the coplanar one. We suppose there is some similar reason in the $i_{\mathrm{m}}=46^{\circ}$ case. As in this situation the first order contributions almost disappear, whereas the small higher order terms can also be more significant. Furthermore, numeric integrations in this case show a disappearence of the identity between the $g_{1}, g_{1}+180^{\circ}$ initial conditions, which also suggests a significance of the higher order terms, because in these terms we can expect the appearance of trigonometric functions with $g_{1}$ and $3 g_{1}$ in their arguments.

As expected, the highly eccentric-distant-companion scenario produces the largest amplitude TTV, at least when a complete revolution is considered. Nevertheless, on a shorter time scale, the length of the observing window necessary for the detection depends highly on the phase of the curve. To illustrate this, in Fig. 6 we plotted the first, and second 8 years of the three primary transit $\mathrm{O}-\mathrm{C}$ curves for both the coplanar, and the perpendicular cases, shown in the first and last rows of Fig. 5. The transiting periods for each curve were calculated in the usual observational manner; i.e., the time interval between the first (some) transits were used. In the $e_{2}=0.7$ cases, according to Fig. 6, it would be unlikely to detect the perturbations within the first eight years, despite that they show the largest (total) amplitude. The same conclusion can be drawn for the coplanar $e_{2}=0.3$ case. The most certain detection would be possible in the two smallest amplitude, circular $e_{2}=0$ configurations. 
T. Borkovits et al.: Transit timing variations in eccentric hierarchical triple exoplanetary systems. I.
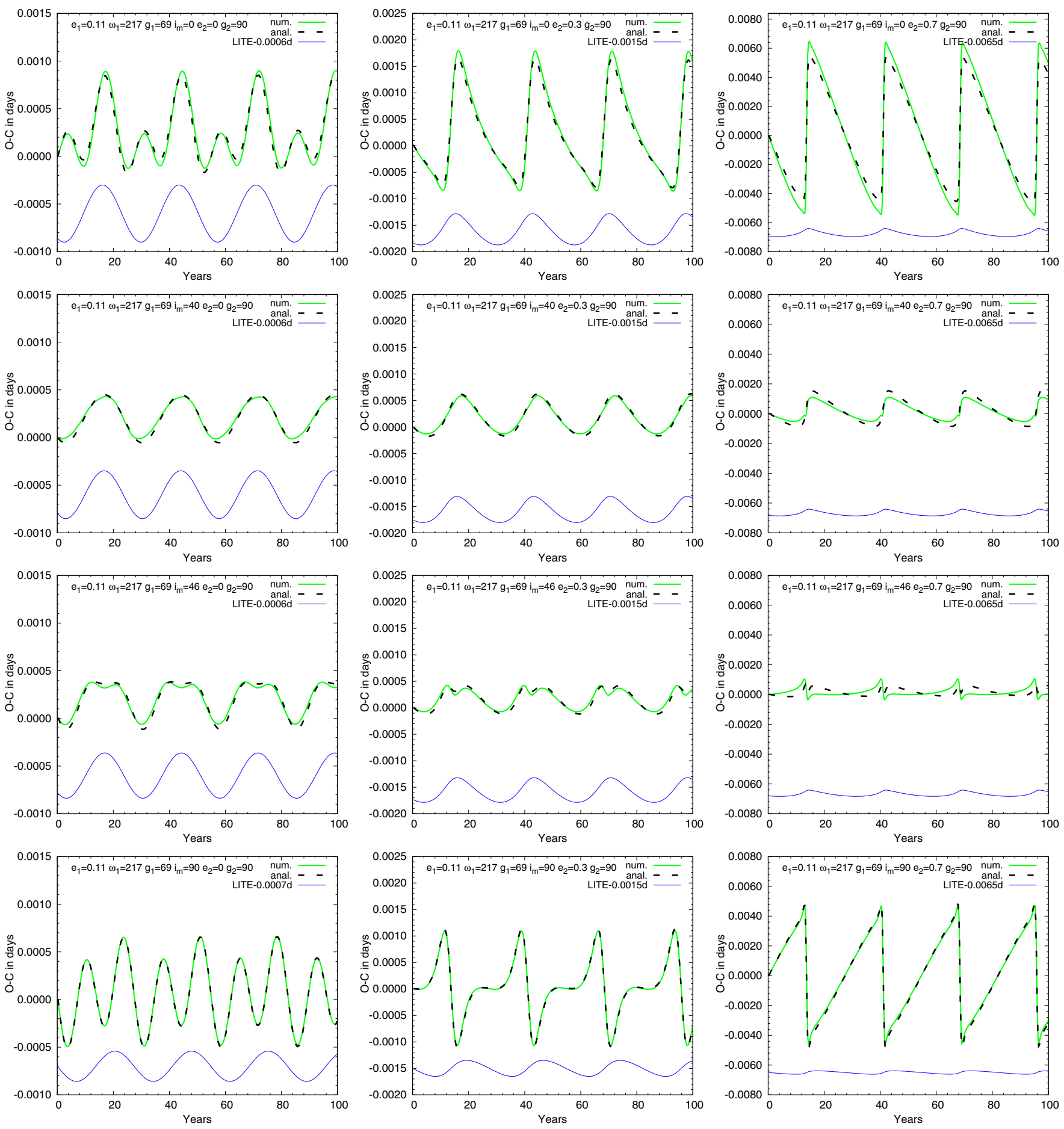

Fig. 5. Sample of transit timing variations caused by a hypothetical $P_{2}=10000$ day-period $m_{3}=0.005 M_{\odot}\left(\approx 5 M_{\mathrm{J}}\right)$ mass third companion for CoRoT-9b at four different initial mutual inclinations $\left(i_{\mathrm{m}}=0^{\circ}, 40^{\circ}, 46^{\circ}, 90^{\circ}\right.$, from up to down), and three different initial outer eccentricities $\left(e_{2}=0,0.3,0.7\right.$, from left to right). The dynamical (relative) arguments of periastrons are set to $g_{1}=69^{\circ}, g_{2}=90^{\circ}$. The curves show the sum of the geometrical LITE, and the dynamical terms obtained both from numeric integrations, and analytic calculations up to sixth order in $e_{1}$. The pure LITE contributions are also plotted separately. Note that the vertical scale of the individual columns (i.e. different $e_{2}$-s) are different.

Nevertheless, if the observations start at those phases plotted in the right panels (8-16 years), the pictures completely differ. In this latter interval, the circular cases produce the smallest curvature $\mathrm{O}-\mathrm{C}-\mathrm{s}$, and the discrepancy from the linear trend reaches 0.001 days (which can be considered as a limit for certain detection) occurring towards the end of the interval. On the other hand, in the case of the highly eccentric configurations, the "moment of truth" comes after some years. Nevertheless, we have to stress that, although we plotted the $\mathrm{O}-\mathrm{C}$ curves with continuous lines, in reality they would contain only 3-4 points during the phase of the seemingly abrupt jump, which is a further complicating factor with regards to "certain" detection.

In the sample runs above, a moderately hierarchic scenario $a_{2} \approx 22 a_{1}$ was studied. To get some picture about the lower limit of the validity of our low-order, hierarchical approximation, we carried out other integrations for less hierarchic configurations. 

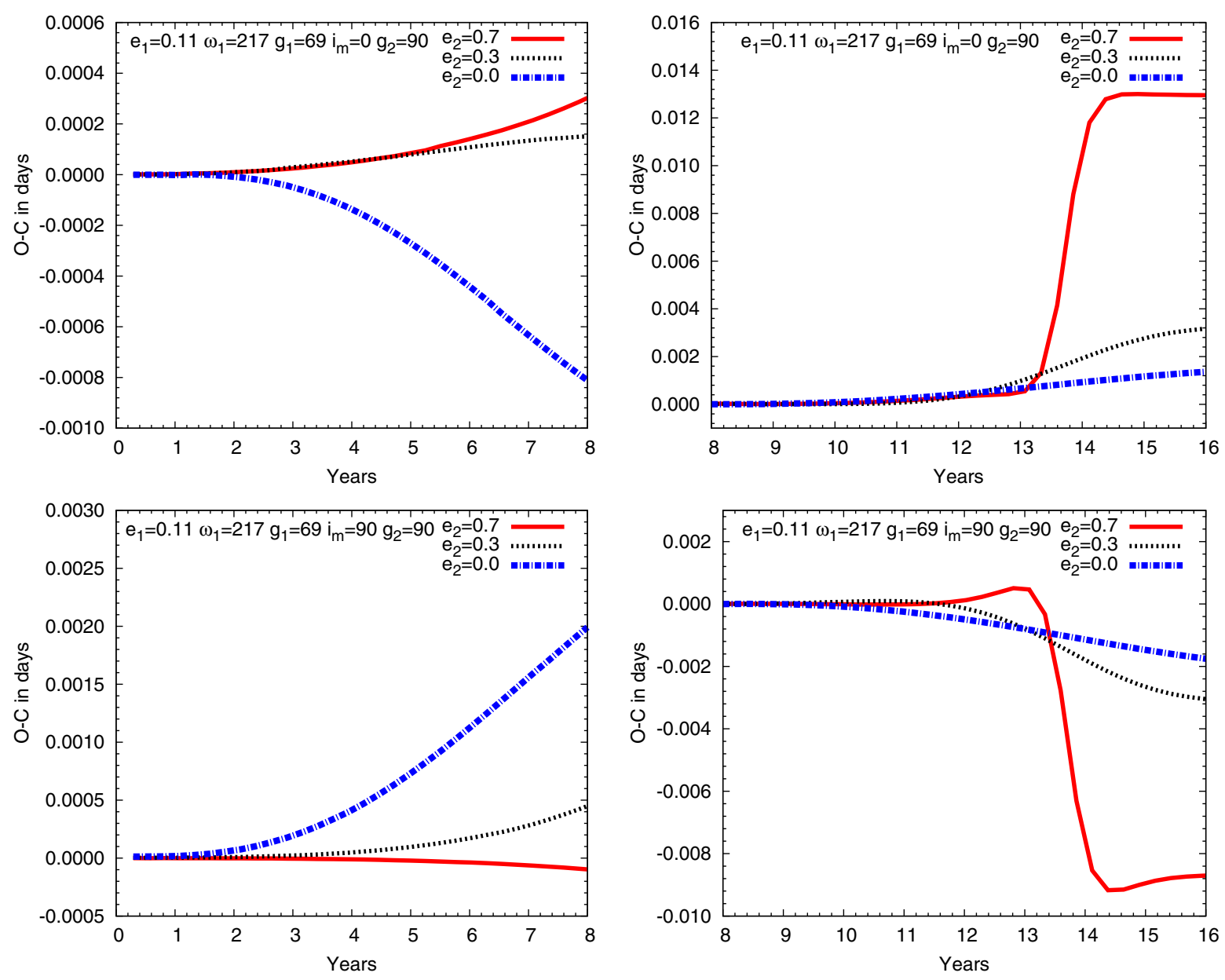

Fig. 6. The first and the second 8 years of $\mathrm{O}-\mathrm{C}$-s plotted in the first and the last rows of Fig. 5. The periods of the individual curves were set equal to the respective initial transiting periods.

In Fig. 7 we show the results of some of these runs, which were carried out with the same initial conditions as used in the middle panel of the first and last rows (coplanar and perpendicular cases, respectively) of Fig. 5, but for $P_{2}=1000$ days (i.e. $a_{2} \approx 4.8 a_{1}$ ), $P_{2}=2000$ days $\left(a_{2} \approx 7.6 a_{1}\right)$, and $P_{2} \approx 952.7$ days $\left(a_{2} \approx 4.6 a_{1}\right)$, in which last case the two planets orbit in 1:10 mean motion resonance. In the left panels of Fig. 7 we plot 20-year-long intervals, while in right ones show century-long time scales. As one can see, on this time scale, apse-node effects already reach or exceed the magnitude of the long period ones. This naturally arises from the fact that the typical time scales of these high-amplitude perturbations are proportional to $P_{2}^{2} / P_{1}$; i.e., in the present cases these are $\sim 100 \times$ faster than in the previously investigated case. For the sake of a better comparison, the analytical curves in the right panels were calculated by including these apse-node terms, although these terms will only be presented in a forthcoming paper. Turning back to the 20-year-long integrations, one can see that the limit of the validity of the present approximation indeed strongly depends on the mutual inclination. While for the $i_{\mathrm{m}}=90^{\circ}$ configurations the long period analytical results are in remarkably good agreement with the numerical curves even for $a_{2}<5 a_{1}$ (left panels of third and forth rows), for the coplanar $\left(i_{\mathrm{m}}=0^{\circ}\right)$ case our approximation is clearly insufficient for such low $a_{2} / a_{1}$ ratios, and even for the doubled outer period case (i.e. $a_{2} \approx 8 a_{1}$ ) the amplitude of the analytical curve is highly underestimated.

We also investigated the case of the 1:10 mean-motion resonance. Our results for the perpendicular case are plotted in the last row. In this case the numerical integration shows very high amplitude apse-node scale variations that do not occur in the analytical curve. To better compare the analytical and numerical long-term variations in this case, we removed the apse-node effect from the numerical curve by using a quadratic term; i.e., the (blue) $\mathrm{O}-\mathrm{C}$ curve was calculated in the form of

$\mathrm{O}-\mathrm{C}=c_{0}+c_{1} E+c_{2} E^{2}$,

where $E$ is the cycle number. As one can see, this quadratic (blue) curve shows similar agreement with the analytical curve, which was found in the similar $a_{2} / a_{1}$ ratio non-resonant case. Consequently, we can state that our long term formulae are capable of producing the same accuracy even around mean-motion resonances.

Nevertheless, from these few arbitrary trial runs we cannot make general statements about the limits of our approximations. A detailed discussion of this point is postponed to a forthcoming paper, where we will include the apse-node time scale terms.

While CoRoT-9b served as an illustration for the TTVs in the low inner eccentricity case, our next sample exoplanet HD 80606b represents the extremely eccentric case.

\section{2. $H D 80606 b$}

The high-mass gas giant exoplanet HD 80606b features an almost 4-month long period and an extremely eccentric orbit around its solar-type host star. It was discovered spectroscopically by Naef et al. (2001). Recently both secondary occultation 
T. Borkovits et al.: Transit timing variations in eccentric hierarchical triple exoplanetary systems. I.
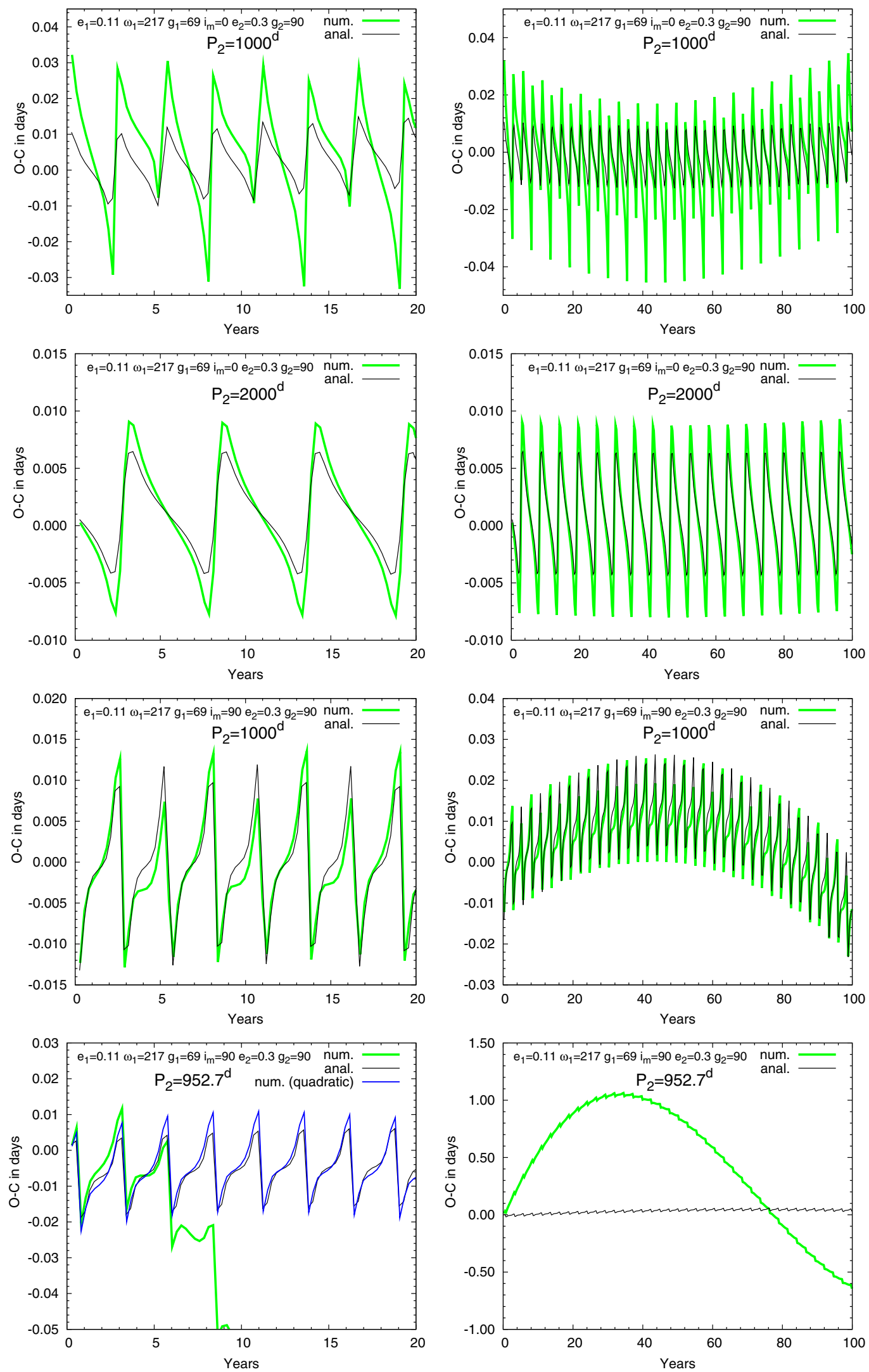

Fig. 7. Checking the validity of hierarchical approximation for closer systems. In the first two rows the initial conditions were set to be the same as at the uppermost middle panel of Fig. 5, with the exception of $P_{2}=1000$ (first row) and 2000 days (second row), i.e. $a_{2} / a_{1} \approx 4.8$ and $\approx 7.6$, respectively, while the third and last rows have initial conditions similar to the bottom middle panel of Fig. 5 , with the exception of $P_{2}=1000$ (third row) and 952.7 days (last row). This latter illustrates the case of a 1:10 mean-motion resonance. The left panels represent a 20-year-long time scale, while the right ones show the TTV behaviour during a century. In the left panel of the last row the blue (quadratic) curve shows the $\mathrm{O}-\mathrm{C}$ curve calculated by including a quadratic term. See text for details. 
(with the Spitzer space telescope, Laughlin et al. 2009), and primary transit (Moutou et al. 2009; Fossey et al. 2009) have been detected. A thorough analysis of the collected data around the February 2009 primary transit led to the conclusion that there is a significant spin-orbit misalignment in the system, i.e. the orbital plane of HD 80606b fails to coincide with the equatorial plane of its host star (Pont et al. 2009). These facts suggest that this planet might be seen at an instant close to the maximum eccentricity phase of a Kozai cycle induced by a distant, inclined third companion (cf. Wu \& Murray 2003; Fabrycky \& Tremaine 2007). Although HD 80606 itself forms a binary with HD 80607 , due to the large separation, one may expect an additional, not so distant companion for an effective Kozai mechanism. The very distant companion HD 80607 may nevertheless play an indirect role in the initial triggering of the Kozai mechanism, by the effect described in Takeda et al. (2009). The most important parameters of the system are summarized in Table 1 .

Due to its very high eccentricity, the periastron distance of this planet is $q_{1}=a_{1}\left(1-e_{1}\right) \approx 0.03 \mathrm{AU}$, at which distance the tidal forces and especially tidal dissipation should be effective. (As a comparison we note that this separation corresponds to the semi-major axis of an approx. 2 day period orbit.) Furthermore, we can also expect a significant relativistic contribution to the apsidal motion. Although such circumstances do not invalidate the following conclusions (as the time scales of these effects are significantly longer than the ones investigated), we nevertheless provide a quantitive estimation of their effects. Furthermore, the effect of dissipation will also be considered numerically at the end of this section.

The apsidal advance speed, averaged for one orbital revolution, can be written in the following form:

$\dot{g}_{1}=A+B \cos 2 g_{1}$,

where the non-zero contributions of the third-body, tidal, and relativistic terms are as follows:

$$
\begin{aligned}
A_{3^{\text {rd }}} & =A_{\mathrm{L}} \frac{P_{1}}{P_{2}}\left(1-e_{1}^{2}\right)^{-1 / 2}\left[I^{2}-\frac{1}{5}\left(1-e_{1}^{2}\right)+\frac{2}{5}\left(1+\frac{3}{2} e_{1}^{2}\right) \frac{C_{1}}{C_{2}} I\right], \\
B_{3^{\text {rd }}} & =A_{\mathrm{L}} \frac{P_{1}}{P_{2}}\left(1-e_{1}^{2}\right)^{-1 / 2}\left[\left(1-e_{1}^{2}\right)-I^{2}-e_{1}^{2} \frac{C_{1}}{C_{2}} I\right] \\
A_{\text {tidal }} & =\frac{5 \mathcal{T}}{2 a_{1}^{5}} \frac{1+\frac{3}{2} e_{1}^{2}+\frac{1}{8} e_{1}^{4}}{\left(1-e_{1}^{2}\right)^{5}}+\frac{\mathcal{R}}{a_{1}^{2}\left(1-e_{1}^{2}\right)^{2}}, \\
A_{\text {rel }} & =6 \pi \frac{G m_{1}}{c^{2} a_{1}\left(1-e_{1}^{2}\right)},
\end{aligned}
$$

where

$$
\begin{aligned}
C_{1} & =\frac{m_{1} m_{2}}{m_{12}} \sqrt{G m_{12} a_{1}\left(1-e_{1}^{2}\right)}, \\
& =L_{1} \sqrt{1-e_{1}^{2}}, \\
C_{2} & =\frac{m_{12} m_{3}}{m_{123}} \sqrt{G m_{123} a_{2}\left(1-e_{2}^{2}\right)}, \\
& =L_{2} \sqrt{1-e_{2}^{2}},
\end{aligned}
$$

moreover,

$$
\begin{aligned}
\mathcal{T} & =6\left(\frac{m_{2}}{m_{1}} k_{2}^{(1)} R_{1}^{5}+\frac{m_{1}}{m_{2}} k_{2}^{(2)} R_{2}^{5}\right), \\
& =6 \frac{m_{2}}{m_{1}} R_{1}^{5}\left[k_{2}^{(1)}+k_{2}^{(2)}\left(\frac{\bar{\rho}_{1}}{\bar{\rho}_{2}}\right)^{2} \frac{R_{1}}{R_{2}}\right], \\
\mathcal{R} & =\frac{k_{2}^{(1)} R_{1}^{5} \omega_{z_{1}^{\prime}}^{2}}{G m_{1}}+\frac{k_{2}^{(2)} R_{2}^{5} \omega_{z_{2}^{\prime}}^{2}}{G m_{2}} \\
& =\frac{3 k_{2}^{(1)}}{4 \pi G \bar{\rho}_{1}} v_{\mathrm{e}_{1}}^{2}+\frac{3 k_{2}^{(2)}}{4 \pi G \bar{\rho}_{2}} v_{\mathrm{e}_{2}}^{2} .
\end{aligned}
$$

In these equations $m_{1}, R_{1}, \bar{\rho}_{1}, k_{2}^{(1)}, \omega_{z_{1}^{\prime}}$, and $v_{\mathrm{e}_{1}}$ refer to the mass, radius, average density, first apsidal motion constant, uni-axial rotational angular velocity, and equatorial rotational velocity of the host star, respectively, while subscript ${ }_{2}$ denotes the same quantities for the inner planet. Also, the rotational term is only valid for non-aligned rotation, and consequently only provides a crude estimation for HD 80606b. Nevertheless, for the present purpose it seems satisfactory. First consider the third-body term. Substituting $\cos 2 g=-1, I^{2}=3 / 5$,

$$
\begin{aligned}
\dot{g}_{3^{\text {rd }}}= & \frac{3 \pi}{2} \frac{m_{3}}{m_{123}}\left(\frac{P_{1}}{P_{2}}\right)^{2}\left(1-e_{2}^{2}\right)^{-3 / 2}\left(1-e_{1}^{2}\right)^{-1 / 2} \\
& \times\left[3 e_{1}^{2} \pm \sqrt{\frac{3}{5}}\left(1+4 e_{1}^{2}\right) \frac{L_{1}}{L_{2}} \sqrt{\frac{1-e_{1}^{2}}{1-e_{2}^{2}}}\right]
\end{aligned}
$$

from which the last term is usually omittable in hierarchical systems, since $C_{1} \ll C_{2}$, which is more expressively true for high $e_{1}$. For example, in the present situation

$\frac{C_{1}}{C_{2}} \approx 0.06\left(1-e_{2}^{2}\right)^{-1 / 2}$

so for HD $80606 \mathrm{~b}$ we find that

$\Delta g_{3^{\text {rd }}} \approx 0.43 \times\left(1-e_{2}^{2}\right)^{-3 / 2}$ century $^{-1}$.

(This result is in excellent correspondence with Fig. 11.)

There are several uncertainties in the calculation of the tidal contribution. While the $k_{2}$ constant is relatively well-known for ordinary stars, it is very ambigous for exoplanets. Furthermore, we know nothing about the rotational velocity of HD 80606b. For the numeric integrations, according to the tables of Claret \& Gimènez (1992) we thus set $k_{2}^{(1)}=0.02$ for the host star, and assume $k_{2}^{(2)}=0.2$ for its planet, which is the same order of magnitude as for Jupiter and for WASP-12b (Campo et al. 2011). The stellar rotation is set to $V_{\text {rot }}=1.8 \mathrm{~km} \mathrm{~s}^{-1}$ (i.e. $P_{\text {rot }}=27.5 ; \omega_{z_{1}^{\prime}}=0.228 \mathrm{day}^{-1}$ ) (Fischer \& Valenti 2005), while for the planet we take (arbitrarily) a one-day rotation period, i.e. $\omega_{z_{2}^{\prime}}=6.283 \mathrm{day}^{-1}$. With these values, the classical tidal contribution to the apsidal motion becomes

$\Delta g_{\text {tidal }} \approx 0.007$ century $^{-1}$,

which can be neglected.

Finally, the relativistic contribution is estimated to be

$\Delta g_{\text {rel }} \approx 0.06$ century $^{-1}$,

i.e. it is smaller by one magnitude than the third-body term, so does also not play any important role. 
T. Borkovits et al.: Transit timing variations in eccentric hierarchical triple exoplanetary systems. I.
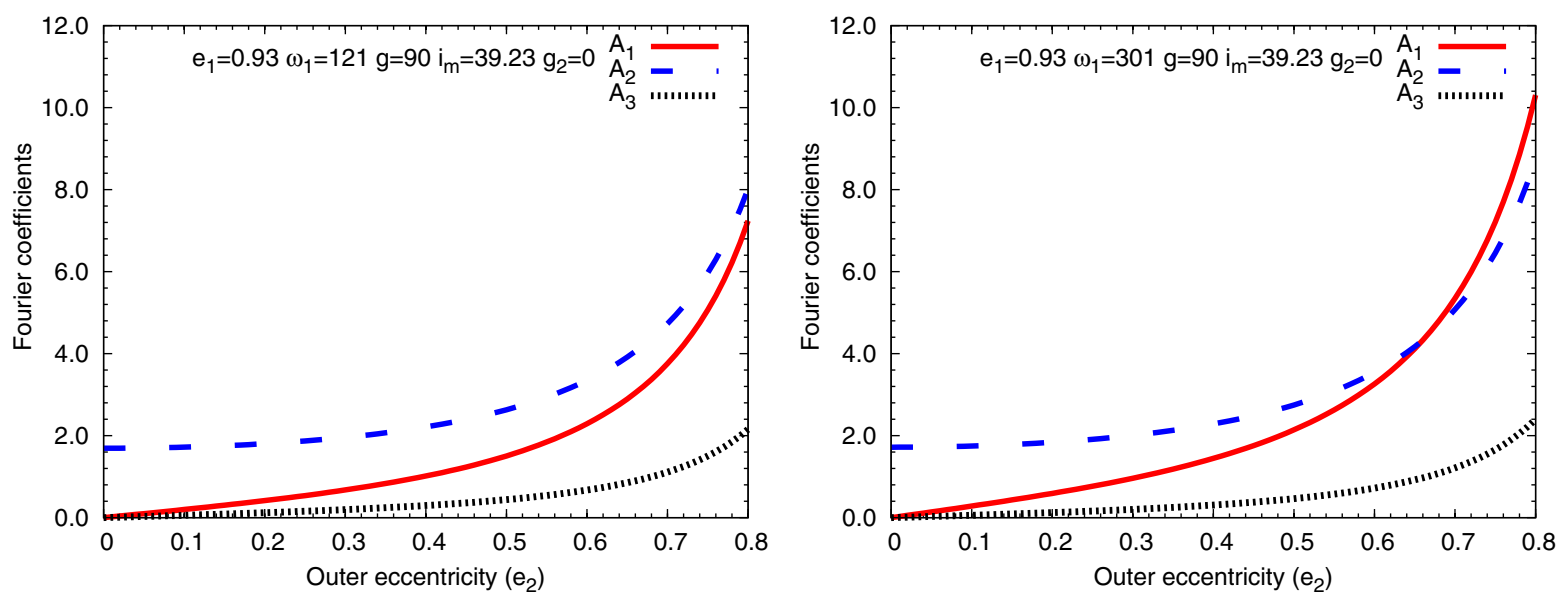

Fig. 8. The $A_{1,2,3}$ amplitudes for HD $80606 \mathrm{~b}$ for primary transits (left), and secondary occultations (right), supposing that the planet is seen at the instant of the maximum phase of a Kozai-cycle.

Returning to the $P_{2}$ time scale variations of the TTVs, we carried out our calculations and integration runs with the supposition that a second, similar mass giant planet is the source of this comet-like orbit, which is seen at the instant of the maximum eccentricity phase of the Kozai-cycle. Consequently, we set $g_{1}=90^{\circ}$, and $i_{\mathrm{m}}=39^{\circ} .23$. With these values from the sixthorder formula, we get $A_{\mathcal{M}}=-0.38, A_{\mathcal{S}}=1.69$ for primary transits, and $A_{\mathcal{M}}=-1.06, A_{\mathcal{S}}=1.72$ for secondary occultations. Consequently, for primary transits, the $\mathrm{O}-\mathrm{C}$ is evidently dominated by the $\mathcal{S}$-term. (The negative $A_{\mathcal{M}}$ indicates a simple $180^{\circ}$ phase shift.) In Fig. 8 the $A_{1,2,3}$ amplitudes are plotted as a function of the outer eccentricity $\left(e_{2}\right)$. Due to the more than four and a half-times larger primary transit $\mathcal{S}$ amplitude, the $g_{2}$ dependence of the amplitudes here are weak, and therefore we show only $A_{1,2,3}$-s for $g_{2}=0^{\circ}$. In Fig. 9 we present both the numerically generated short-term $\mathrm{O}-\mathrm{C}$ curves and the analytically calculated cases up to the sixth order in eccentricity (see Appendix B) for three different eccentricities $\left(e_{2}=0,0.3,0.7\right)$ of the outer perturber's orbit. We plotted the O-C-s for both primary transits and secondary occultations. Also shown are the corresponding primary minus secondary curves.

As one can see, the sixth-order formulae gave satisfactory results even for such high eccentricities, although the analytical amplitudes are somewhat overestimated. Nevertheless, a more detailed analysis shows that the accuracy of our formulae for such high inner eccentricities strongly depends on the other orbital parameters. This is illustrated even in the present situation, where, for the secondary-occultation curves, (which correspond to $\omega_{1}=301^{\circ}$ ), the discrepancies are clearly larger. From this point of view, the $e_{2}=0$ case (first row) is even more interesting, because in this situation, where the only one non-vanishing amplitude is $A_{2}=A_{\mathcal{S}}$, the similar $A_{\mathrm{S}}$ values would lead to almost identical $\mathrm{O}-\mathrm{C}$ curves (compare the corresponding dashed analytical curves). However, according to the numerical results (see the solid curves), this, in fact, is not the case.

Considering the case of fastest possible detection of the amplitudes, in Fig. 10 we also plotted the first and second 8 years of the three primary transit $\mathrm{O}-\mathrm{C}$ curves shown in Fig. 9. The transiting periods for each curves were calculated in the usual observational manner, i.e. with the time interval between the first (some) transits. According to Fig. 10, in the first eight years, i.e. after the apastron of the outer body, the fastest detection would be possible in the smallest (total) amplitude circular third body case, while the curvature of the highly eccentric curve is so small that it needs almost seven years to exceed the 0.001 difference, which could promise certain detection. (Of course, this is a non-realistic ideal case, where all the transits are measured without any observational error.) Around periastron (right panel), the situation is completely different, similar to the case of CoRoT-9b.

We also carried out numerical integrations to investigate the possible orbital evolution of HD $80606 \mathrm{~b}$ in the presence of such a perturber. We have shown that both relativistic and tidal effects can be omitted in the present configuration of the system. However, this assumption is only correct in those cases where tidally forced dissipation is not considered. Consequently, we have integrated the dynamical evolution of the system both with tidal effects (both dissipative and conservative tidal terms) and without them (i.e. in the frame of pure three, point-mass gravitational interactions). The equations of the motion (including tidal and dissipative terms and stellar rotation) are given in Borkovits et al. (2004), where the description of our integrator can also be found. In the dissipative case, our dissipation constant was selected in such a way that it produced $\Delta t_{1} \approx 2.16 \times 10^{-7}$ tidal lag-time for the host-star, and $\Delta t_{1} \approx$ $5^{\mathrm{d}} .21 \times 10^{-4}$ for the planet, which are equivalent to $Q_{1} \approx 4.1 \times 10^{7}$, $Q_{2} \approx 1.7 \times 10^{4}$ dissipation parameters, respectively. Figure 11 shows the variation in most of the orbital elements (both dynamical and observational) for 100 and 1 million years in the dynamically most-excited $e_{2}=0.7$ case. Thin lines represent the dissipative case, while thick curves show the point-mass result. The century-long left panel demonstrates that, apart from the shrinking semi-major axis, there is no detectable variation in the orbital elements during such a short time interval. And, of course, if this is true for the extremum of the Kozai cycle, it is more expressly valid for other situations, as this phase produces the fastest orbital element variations. Furthermore, on such a short time scale, the orbital variations in a point-mass or nonKeplerian, tidal framework are indistinguishable. (Again, we do not take the semi-major axis into account.) This provides further verification of the effects discussed previously, namely that we have neglected the tidal and relativistic effects completely and considered all the orbital elements as constant.

Now, we consider orbital shrinking due to dissipation. As one can see, in the present situation the decrease in $a_{1}$ during the first 100 years is $\Delta a_{1} \approx 6 \times 10^{-4} R_{\odot}$. Converting this 

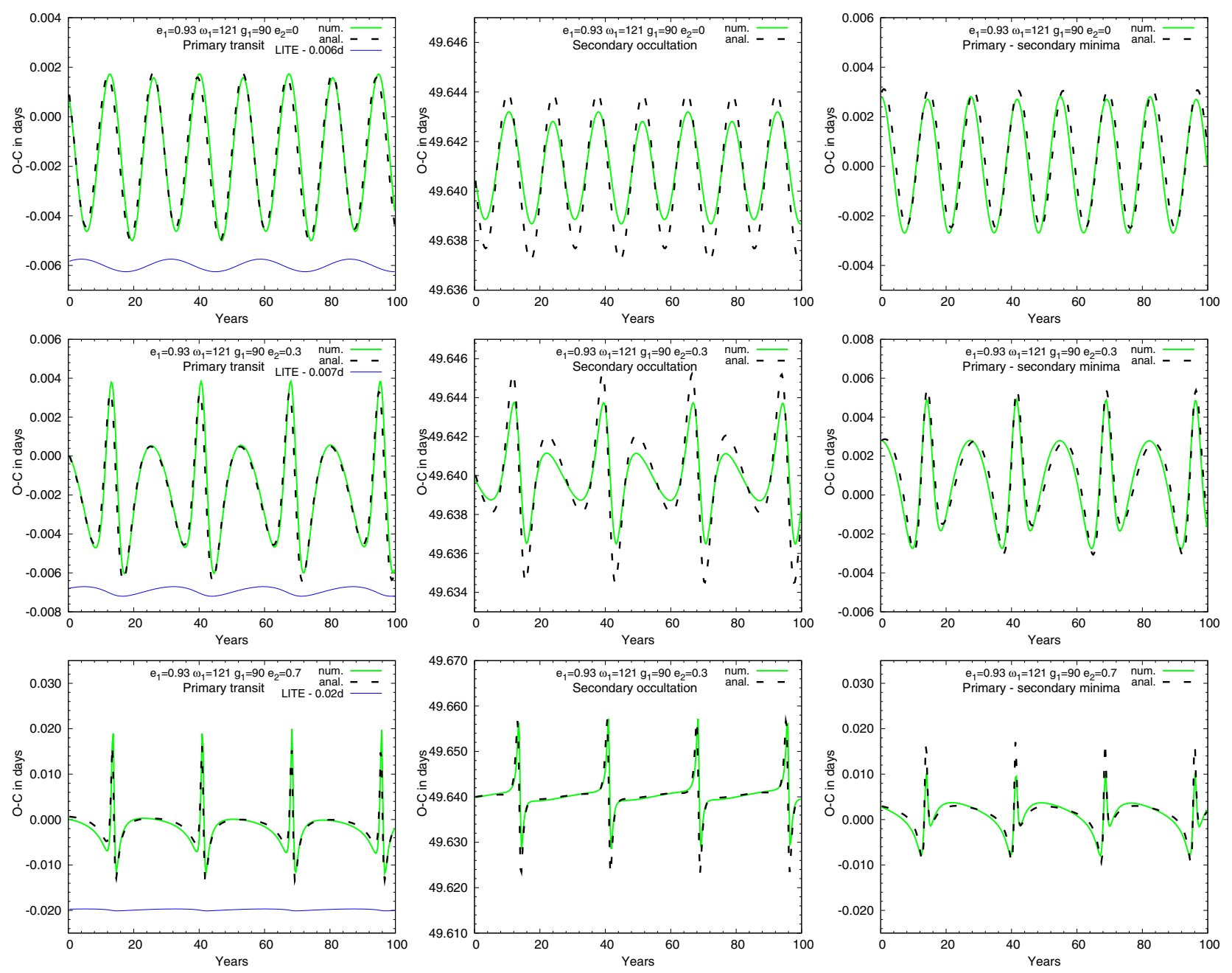

Fig. 9. Transit timing variatons caused by a hypothetical $P_{2}=10000$ day-period $m_{3}=0.005 M_{\odot}\left(\approx 5 M_{\mathrm{J}}\right)$ mass third companion for HD $80606 \mathrm{~b}$ at the maximum eccentricity phase of the induced Kozai-cycles. Top: $e_{2}=0 ;$ middle: $e_{2}=0.3$; bottom: $e_{2}=0.7\left(g_{2}=0^{\circ}\right.$ in all cases); left panels: primary transits; middle panels: secondary occultations; right panels: primary minus secondary difference. See text for further details. (For better comparison the curves are corrected for the different average transit periods, and zero point shifts.)

into a period variation suggests $\Delta P_{1} \approx 10^{-3} \mathrm{~d}$. This gives a $\dot{P}_{1} \approx 3 \times 10^{-6}$ day cycle ${ }^{-1}$ rate for one transiting period. From the point of view of an eclipsing binary observer, this is an incredibly high value. For comparison, a typical, secular period variation rate measured for many of the eclipsing binaries is about a $10^{-9}-10^{-11}$ day cycle ${ }^{-1}$. Such a high rate would produce 0.001 departure in transit time during $\sim 26$ cycles, i.e. during approximately eight years. This period variation ratio is close to the ratio produced by typical long-term perturbations within a few years. This illustrates that, if the data-length is significantly shorter than the period of the long-term periodic perturbations, then the effect has arisen from such perturbations, and other effects of i.e. orbital shrinking can overlap each other and can as a result, be misinterpreted easily. (The question of such kinds of misinterpretations or false identifications were considered in general in Sect. 2 of Borkovits et al. 2005.)

Finally, we consider the right panel of Fig. 11, which illustrates the frequently mentioned Kozai-mechanism (with and without tidal friction) in operation. In the present situation, because of the high outer eccentricity and along with the relatively weak hierarchicity of the system (i.e. $a_{1} / a_{2} \approx 0.05$ ), the higher order terms of the perturbation function are also significant, which results in very different consecutive cycles even in the point-mass case. This manifests itself not only as different periods and maximum eccentricities, but also in how the argument of (dynamical) periastron $\left(g_{1}\right)$ shows both circulation and libration, alternately (cf. Ford et al. 2000). Nevertheless, the detailed investigation of the apse-node time scale behaviour of the TTV, as well as other orbital elements and observables, will be the subject of a subsequent paper.

\section{Conclusions}

We have studied the long-term $P_{2}$ time scale TTVs in transiting exoplanetary systems, which feature an additional, more distant $\left(a_{2} \gg a_{1}\right)$ planetary, or else stellar, companion. We gave the analytical form of the $\mathrm{O}-\mathrm{C}$ diagram, which describes such TTVs. Our result is an extension of our previous work Borkovits et al. (2003) for arbitrary orbital elements of both the inner transiting planet and the outer companion. We showed that the dependence of the $\mathrm{O}-\mathrm{C}$ on the orbital and physical parameters can be separated into three parts. Two of these are independent of the real physical parameters (i.e. masses, separations, periods) of a concrete system and only depend on dimensionless orbital elements, and so can be analysed in general. For the two other kinds of parameters, which are amplitudes and phases of trigonometric 
T. Borkovits et al.: Transit timing variations in eccentric hierarchical triple exoplanetary systems. I.
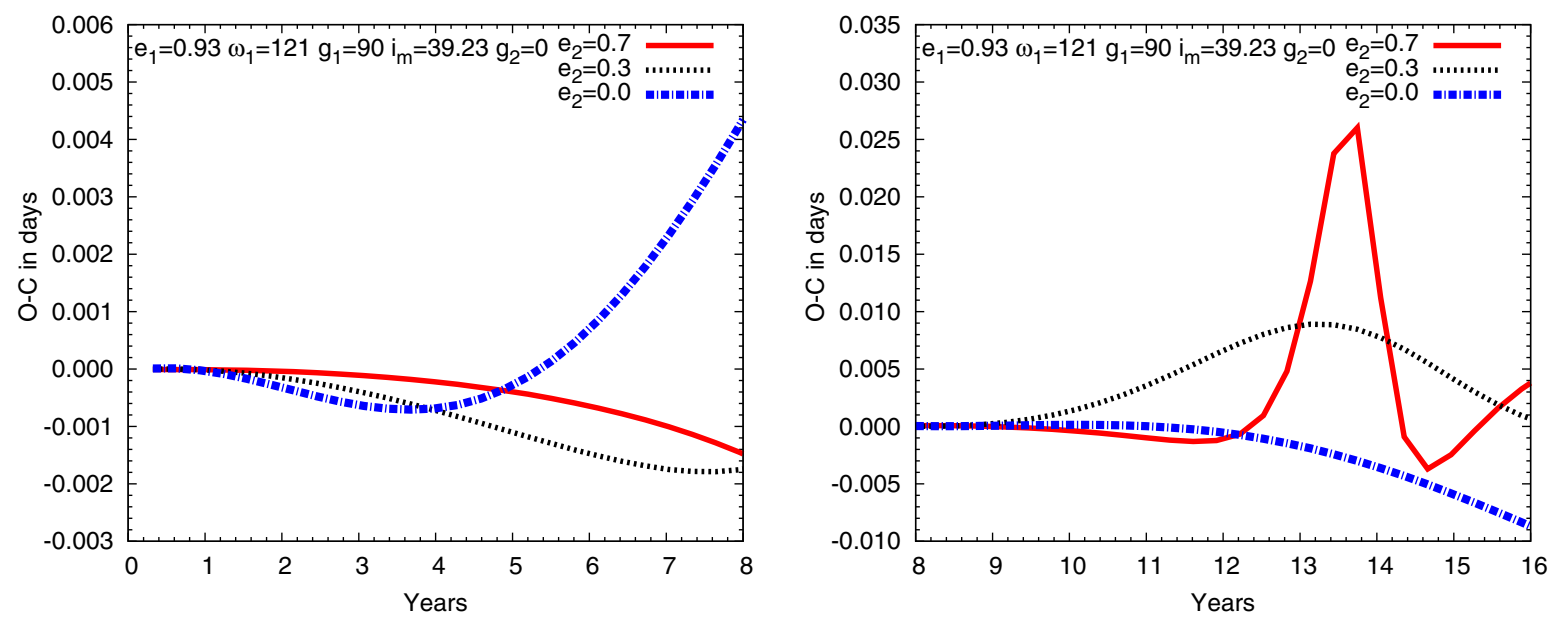

Fig. 10. The first and second 8 years of the left panels of Fig. 9. The periods of the individual curves was set equal to the each initial transiting periods.

functions, we separated the orbital elements of the inner from the outer planet. The practical importance of such a separation is that in the case of any actual transiting exoplanets, if eccentricity $\left(e_{1}\right)$ and the observable argument of periastron $\left(\omega_{1}\right)$ are known, e.g., from spectroscopy, then the main characteristics of any, caused by a possible third body, TTVs can be mapped simply by the variation in two free parameters (dynamical, relative argument of periastron, $g_{1}$, and mutual inclination, $i_{\mathrm{m}}$ ), which then can be refined by the use of the other derived parameters, including two additional parameters for the possible third body (eccentricity, $e_{2}$, and dynamical, relative argument of periastron, $g_{2}$ ). Moreover, as the physical attributes of a given system only occur as scaling parameters, the real amplitude of the $\mathrm{O}-\mathrm{C}$ can also be estimated for a given system, simply as a function of the $m_{3} / P_{2}$ ratio.

At this point it would be beneficial to compare our results with the conclusions of Nesvorný \& Morbidelli (2008) and Nesvorný (2009). These authors have investigated the same problem, i.e. the fast detection of outer perturbing planets and the determination of their orbital and physical parameters from their perturbations on the transit timing of the inner planet, by the help of the analytical description of the perturbed transiting $\mathrm{O}-\mathrm{C}$ curve. For the mathematical description, they use the explicit perturbation theory of Hori (1966) and Deprit (1969), based on canonical transformations and on the use of Lie-series. This theory does not require the hierarchical assumption; i.e., the $\alpha=a_{1} / a_{2}$ parameter, although less than unity, is not required to be small. This suggests a somewhat greater generality of the results, so it is applicable systems similar to our solar system. Nevertheless, perhaps a small disadvantage to this method over the hierarchical approximation is that, for large mutual inclinations, the number of terms in the perturbation function needed for a given accuracy grows very fast, while our formulae have the same accuracy even for the highest mutual inclinations. There is also a similar discomfort in the case of high eccentricities, in which case the general formulae of Nesvorný (2009) are more sensitive to these parameters than in the hierarchical case. For example, we could reproduce satisfactory accuracy even for $e_{1}=0.9$, in which case the classical formulae are divergent. (Although we concentrate only on the longperiod perturbations, the same is valid for the apse-node time scale variations, as will be illustrated in the next paper.) As a conclusion, the method used by Nesvorný \& Morbidelli (2008) and Nesvorný (2009) beyond the evident non-hierarchical configurations is similarly (or even better) applicable in the nearly coplanar case, too, especially when the inner orbit is nearly circular (a special case where the first-order hierarchical approximation becomes insufficient, see e.g. Ford et al. 2000), but in other cases, our approach could give a faster and simpler method, as long as the hierarchical assumption is satisfied. Furthermore, since in the hierarchical approximation, the principal small parameter in the perturbation equations is the ratio of the separations instead of the mass ratio, these formulae are valid for stellar mass objects as well, and can also be applied to planets orbiting an S-type orbit in binary stars, or even for hierarchical triple stellar systems.

We analysed the above-mentioned dimensionless amplitudes for different arbitrary initial parameters, as well as for two concrete systems CoRoT-9b and HD 80606b. We found in general that, while the shape of the $\mathrm{O}-\mathrm{C}$ strongly varies with the angular orbital elements, the net amplitude (departing from some specific configurations) depends only weakly on these elements, but strongly on the eccentricities. Nevertheless, we found some situations around $i_{\mathrm{m}}=45^{\circ}$ for the specific case of CoRoT-9b, where the $\mathrm{O}-\mathrm{C}$ almost disappeared.

We used CoRoT-9b and HD 80606b for case studies. Both giant planets revolve on several monthly orbits. The former has an almost circular orbit, while the latter has a comet-like, extremely eccentric orbit. These large-period systems are ideal for searching for other perturbing components, since the amplitude of the $\mathrm{O}-\mathrm{C}$ is multiplied by $P_{1}^{2} / P_{2}$ and consequently, as the magnitude of the perturbations determined by $P_{1} / P_{2}$, the same amplitude perturbations cause a more detectable effect, if the characteristic size of the system (i.e. $P_{1}$ ) is larger.

We also considered the questions of both detection and the correct identification of such perturbations. We emphasize again that the $\mathrm{O}-\mathrm{C}$ curve is a very effective tool for detecting any period variations, thanks to its cumulative nature. Nevertheless, some care is necessary. First, it has to be kept in mind that the detectability of a period variation depends on the curvature of the $\mathrm{O}-\mathrm{C}$ curve. (If the plotted $\mathrm{O}-\mathrm{C}$ is simply a straight line, with any given slope, it means that the period is constant, which is known with an error equal to that slope.) This implies that the interval needed to detect the period variation coming from a periodic phenomenon depends more strongly on which phase is observed than on the amplitude of the total variation. (We 

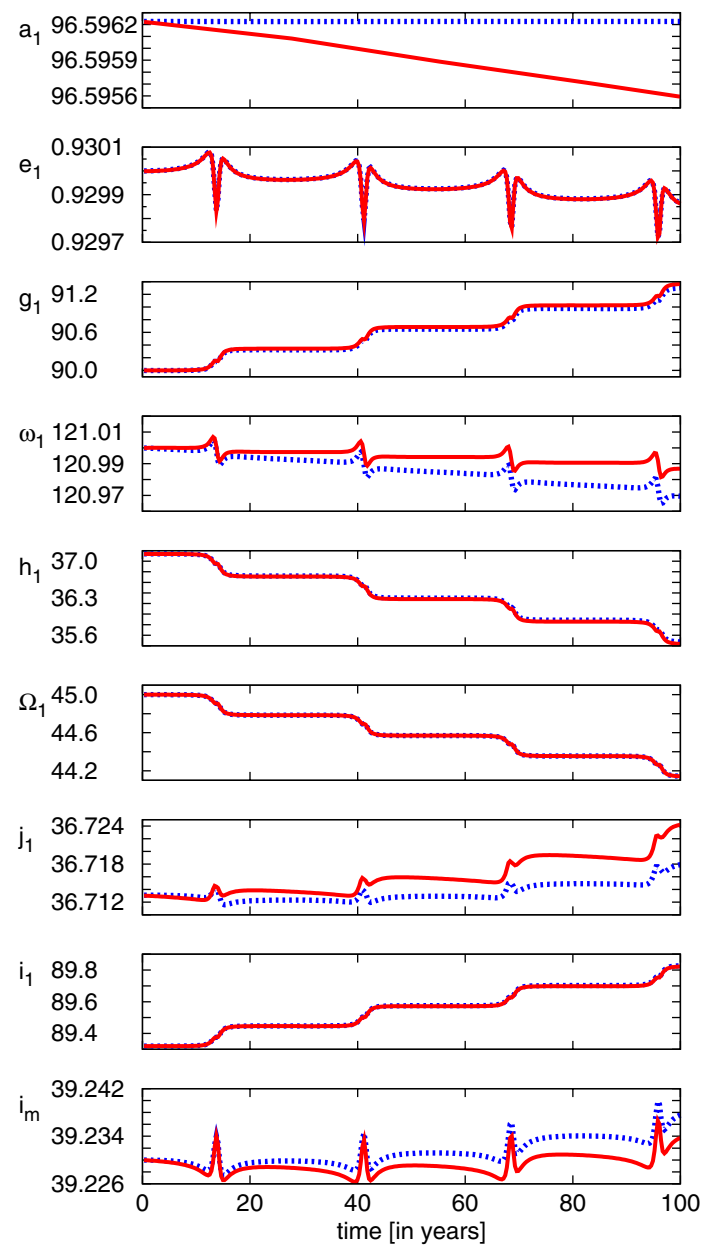
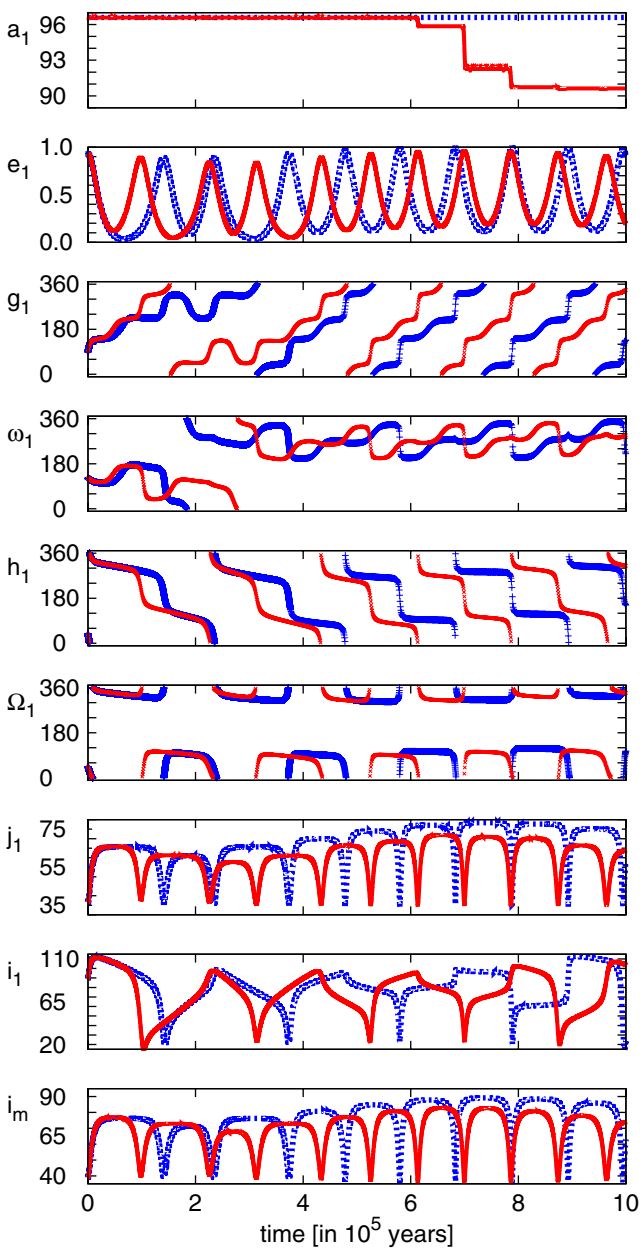

Fig. 11. Dynamical evolution of the orbital elements of HD 80606b in the presence of a hypothetical $P_{2}=10000$ day-period $m_{3}=$ $0.005 M_{\odot}\left(\approx 5 M_{\mathrm{J}}\right)$ mass third companion. The initial orbital elements correspond to the last row of Fig. 9. Red curves: tidal effects and dissipation are considered; blue curves: three point-mass model. (Relativistic contributions are omitted.) Note, the semi-major axis $\left(a_{1}\right)$ is given in $R_{\odot}$.

illustrated this possibility for both systems sampled.) We also illustrated that, in the case of a very eccentric third companion, the fastest rate period variation lasts a very short interval, which consists of only a few transit events. This emphasizes the importance of observing all possible transit (and, of course, occultation) events, with great accuracy. Another question is the possible misinterpretation of the $\mathrm{O}-\mathrm{C}$ diagram. For example, as was shown, in the HD 80606b system we can expect a secular period change due to tidal dissipation. On a short time scale of decades it may happen that this change is of the same order as the one expected from periodic perturbations of a third companion. These two types of perturbations could be separated in two different ways. One way is simply a question of time. In the case of an enough long observing window, a periodic pertubation would separate from a secular one, which would in turn produce a parabola-like $\mathrm{O}-\mathrm{C}$ continuously. But, there is also a faster possibility. In the case that not only primary transits, but also secondary occultations, are observed with similar frequency and accuracy, then, on subtracting the two (primary and secondary) $\mathrm{O}-\mathrm{C}$ curves from each other, the secular change - i.e., the effect of dissipation - would disappear, since it is similar for primary transits and secondary occultations. This also makes it desirable to collect as many occultation observations as possible too.
Acknowledgements. This research made use of NASA's Astrophysics Data System Bibliographic Services. We thank Drs. John Lee Greenfell and Imre Barna Bíró for the linguistic corrections. We thank for the referee and the language editor for their valuable suggestions and comments.

\section{References}

Agol, E., Steffen, J., Sari, R., \& Clarkson, W. 2005, MNRAS, 359, 567 Borkovits, T., Érdi, B., Forgács-Dajka, E., \& Kovács, T. 2003, A\&A, 398, 1091 Borkovits, T., Forgács-Dajka, E., \& Regály, Zs. 2004, A\&A, 426, 951 Borkovits, T., Elkhateeb, M. M., Csizmadia, Sz., et al. 2005, A\&A, 441, 1087 Borkovits, T., Forgács-Dajka, E., \& Regály, Zs. 2007, A\&A, 473, 191 Brown, E. W. 1936, MNRAS, 97, 62

Campo, C. J., Harrington, J., Hardy, R. A., et al. 2011, ApJ, 727, 125

Claret, A., \& Gimènez, A. 1991, A\&AS, 96, 255

Cabrera, J. 2010, EAS Publ. Ser., 42, 109

Chandler, S. C. 1892 , AJ, 11, 113

Cowling, T. G. 1938, MNRAS, 98,734

Deeg, H. J., Ocana, B., Kozhevnikov, V. P., et al. 2008, A\&A, 480, 563

Deeg, H. J., Moutou, C., Erikson, A., et al. 2010, Nature, 464, 384

Deprit, A. 1969, Cel. Mech., 1, 12

Fabrycky, D. 2010, [arXiv: 1006.3834]

Fabrycky, D., \& Tremaine, S. 2007, ApJ, 669, 1298

Fischer, D. A., \& Valenti, J. 2005, ApJ, 622, 1102

Ford, E. B., Kozinsky, B., \& Rasio, F. A. 2000, ApJ, 535, 385

Fossey, S. J., Waldman, I. P., \& Kipping, D. M. 2009, MNRAS, 396, L16

Gimènez, A., \& Garcia-Pelayo, J. M. 1983, Ap\&SS, 92, 203 
T. Borkovits et al.: Transit timing variations in eccentric hierarchical triple exoplanetary systems. I.

Harrington, R. S. 1968, AJ, 73, 190

Harrington, R. S. 1969, Cel. Mech., 1, 200

Hertzsprung, E. 1922, B.A.N., 1, 87

Heyl, J. S., \& Gladman, B. J. 2007, MNRAS, 377, 1511

Holman, M. J. 2010, EAS Pub. Ser., 42, 39

Holman, M. J., \& Murray, N. W. 2005, Science, 307, 1288

Hori, G. 1966, PASJ, 18, 287

Irwin, J. B. 1952, ApJ, 116, 211

Jordán, A., \& Bakos, G. Á. 2008 ApJ, 685, 543

Kiseleva, L. G., Eggleton, P. P., \& Mikkola, S. 1998, MNRAS, 300, 292

Kipping, D. M. 2009a, MNRAS, 392, 181

Kipping, D. M. 2009b, MNRAS, 396, 1797

Kozai, Y. 1962, AJ, 67, 591

Laughlin, G., Deming, D., Langton, J., et al. 2009, Nature, 457, 562

Lee, J. W., Kim, S.-L., Kim, C.-H., et al. 2009, AJ, 137, 3181

Mazeh, T., \& Saham, J. 1979, A\&A, 77, 145

Miralda-Escudé, J. 2002, ApJ, 564, 1019

Moutou, C., Hebrard, G., Bouchy, F., et al. 2009, A\&A, 498, L5

Naef, D., Latham, D. W., Mayor, M., et al. 2001, A\&A, 375, L27

Nesvorný, D., 2009, ApJ, 701, 1116

Nesvorný, D., \& Beaugé, A. 2010, ApJ, 709, L44

Nesvorný, D., \& Morbidelli, A. 2008, ApJ, 688, 636

Pál, A. 2010, MNRAS, 409, 975

Pont, F., Hébrard, G., Irwin, J. M., et al. 2009, A\&A, 502, 695

Qian, S.-B., Liao, W.-P., Zhu, L.-Y., et al. 2009, MNRAS, 401, L34

Sándor, Zs., Süli, Á., Érdi, B., Pilat-Lohinger, E., \& Dvorak, R. 2007, MNRAS, 375,1495

Schwarz, R., Süli, Á., Dvorak, R., \& Pilat-Lohinger, E. 2009, CeMDA, 104, 69

Simon, A., Szatmáry, K., \& Szabó, Gy. M. 2007, A\&A, 470, 727

Silvotti, R., Schuh, S., Janulis, R., et al. 2007, Nature, 449, 189

Söderhjelm, S. 1975, A\&A, 42, 229

Söderhjelm, S. 1982, A\&A, 107, 54

Söderhjelm, S. 1984, A\&A, 141, 232

Sterne, T. E. 1939, MNRAS, 99, 451

Takeda, G., Kita, R., \& Rasio, F. A. 2009, IAU Symp., 253, 181

Tokovinin, A. A. 1997, A\&AS, 124, 75 http://www.ctio.noao.edu/ atokovinin/stars/index.php (continuously refreshed)

Tokovinin, A. A. 2008, MNRAS, 389, 925

Tokovinin, A. A., Thomas, S., Sterzik, M., \& Udry, S. 2006, A\&A, 450, 681

Woltjer, J., Jr. 1922, B.A.N., 1, 93

Wu, Y., \& Murray, N. 2003, ApJ, 589, 605

\section{Appendix A: Relation between the observable, and the dynamical orbital elements.}

First we emphasize that some of the relations between the elements, given in this section, are strictly valid only for the case shown in Fig. 1. According to the actual orientations of the orbital planes, the spherical triangle (with sides $u_{\mathrm{m} 1}, u_{\mathrm{m} 2}, \Omega_{1}-\Omega_{2}$ ) could be oriented in different ways, and so some precise discussion is necessary, which is omitted in the present appendix.

The relation between the pericentrum arguments is

$\omega_{1}=g_{1}+u_{\mathrm{m} 1}$

$\omega_{2}=g_{2}+u_{\mathrm{m} 2}+180^{\circ}$.

Consequently, the true longitudes measured from the sky $(u)$ and from the intersection of the two orbital planes $(w)$ are

$$
\begin{aligned}
u_{1} & =v_{1}+\omega_{1}, \\
u_{2} & =v_{2}+\omega_{2}, \\
w_{1} & =v_{1}+g_{1}, \\
& =u_{1}-u_{\mathrm{m} 1}, \\
w_{2} & =v_{2}+g_{2}+180^{\circ}, \\
& =u_{2}-u_{\mathrm{m} 2} .
\end{aligned}
$$

There are two relations between the mutual inclination $\left(i_{\mathrm{m}}\right)$ and the two dynamical inclinations $\left(j_{1,2}\right)$. One of these is trivial, while the second comes from the relation of the inclinations to the orbital angular momenta. The trivial case is

$i_{\mathrm{m}}=j_{1}+j_{2}$,

while the non-trivial case comes from the fact that

$$
\begin{aligned}
& \cos j_{1}=\frac{\boldsymbol{C} \boldsymbol{C}_{1}}{C C_{1}}, \\
& \sin j_{1}=\frac{\left|\boldsymbol{C} \times \boldsymbol{C}_{1}\right|}{C C_{1}},
\end{aligned}
$$

i.e.

$$
\begin{aligned}
& \cos j_{1}=\frac{C_{1}}{C}+\frac{C_{2}}{C} I, \\
& \sin j_{1}=\frac{C_{2}}{C} \sin i_{\mathrm{m}},
\end{aligned}
$$

where $\boldsymbol{C}_{1,2}$ represents the orbital angular momentum vector of the two orbits, $\boldsymbol{C}$ the net orbital angular momentum vector, and

$$
\begin{aligned}
C_{1} & =\frac{m_{1} m_{2}}{m_{12}} \sqrt{G m_{12} a_{1}\left(1-e_{1}^{2}\right)}, \\
C_{2} & =\frac{m_{12} m_{3}}{m_{123}} \sqrt{G m_{123} a_{2}\left(1-e_{2}^{2}\right)}, \\
C & =C_{1} \cos j_{1}+C_{2} \cos j_{2} .
\end{aligned}
$$

(The rotational angular momenta were neglected.) At this point we notice that for hierarchical systems it is a very reasonable to assume

$C_{1} \cos j_{1}=$ const.,

(since the first-order, doubly averaged Hamiltonian of such systems does not contain its conjugated variable, $h_{1}$ ), which property, together with the constancy of the semi-major axis, connects the eccentricity $\left(e_{1}\right)$ with the dynamical inclination $\left(j_{1}\right)$.

Further relations can be written by the use of the several identities of spherical triangles, for example

$$
\begin{aligned}
\cos i_{\mathrm{m}} & =\cos i_{1} \cos i_{2}+\sin i_{1} \sin i_{2} \cos \left(\Omega_{2}-\Omega_{1}\right), \\
\sin i_{\mathrm{m}} \sin u_{\mathrm{m} 2} & =\sin i_{1} \sin \left(\Omega_{2}-\Omega_{1}\right), \\
\sin i_{\mathrm{m}} \sin u_{\mathrm{m} 1} & =\sin i_{2} \sin \left(\Omega_{2}-\Omega_{1}\right), \\
\sin i_{\mathrm{m}} \cos u_{\mathrm{m} 2} & =-\cos i_{1} \sin i_{2}+\sin i_{1} \cos i_{2} \cos \left(\Omega_{2}-\Omega_{1}\right), \\
\sin i_{\mathrm{m}} \cos u_{\mathrm{m} 1} & =\cos i_{2} \sin i_{1}-\sin i_{2} \cos i_{1} \cos \left(\Omega_{2}-\Omega_{1}\right),
\end{aligned}
$$

and similar ones can be written for the two smaller spherical triangles. Finally, in hierarchical systems, usually $C_{2} \gg C_{1}$, so we can take the following as a first approximation:

$j_{1}=i_{\mathrm{m}}$,

$j_{2}=0$,

$i_{0}=i_{2}$,

$h_{1}=u_{\mathrm{m} 2}$.

In Fig. 1, for practical reasons, we chose the intersection of the invariable plane and the sky for the arbitrary starting point of the observable nodes $\left(\Omega_{1}, \Omega_{2}\right)$. Traditionally, in astrometry, these quantities are measured from north to east on the sky; nevertheless, such a practical choice is valid since the $\Omega$-s only appear in the equations in the form of their differences, and derivatives. (Nodes cannot be determined from photometry and spectroscopy, as both the light curves and the radial velocity curves are invariant for any orientation of the orbital planes projected onto the sky.) 


\section{Appendix B: The derivatives used for calculating the long-period dynamical O-C up to sixth order in $e_{1}$}

The derivatives of the indirect terms are as follows:

$$
\begin{aligned}
\frac{\mathrm{d} e_{1} \cos \omega_{1}}{\mathrm{~d} v_{2}}= & A_{\mathrm{L}}\left(1+e_{2} \cos v_{2}\right)\left\{( 1 - e _ { 1 } ^ { 2 } ) ^ { 1 / 2 } \left\{-\frac{3}{5} e_{1} \sin \omega_{1}\left[\left(I^{2}-\frac{1}{3}\right)+\left(1-I^{2}\right) \cos \left(2 v_{2}+2 g_{2}\right)\right]\right.\right. \\
& -e_{1} \sin \left(\omega_{1}-2 g_{1}\right)\left[\left(1-I^{2}\right)+\left(1+I^{2}\right) \cos \left(2 v_{2}+2 g_{2}\right)\right] \\
& \left.-e_{1} \cos \left(\omega_{1}-2 g_{1}\right) 2 I \sin \left(2 v_{2}+2 g_{2}\right)\right\} \\
& +\frac{\sin i_{\mathrm{m}} \cot i_{1}}{\left(1-e_{1}^{2}\right)^{1 / 2}}\left\{\left[\frac{2}{5}\left(1+\frac{3}{2} e_{1}^{2}\right) \cos u_{\mathrm{m} 1}-e_{1}^{2} \cos \left(2 g_{1}+u_{\mathrm{m} 1}\right)\right] e_{1} \sin \omega_{1} I\left[1-\cos \left(2 v_{2}+2 g_{2}\right)\right]\right. \\
& \left.\left.+\left[\frac{2}{5}\left(1+\frac{3}{2} e_{1}^{2}\right) \sin u_{\mathrm{m} 1}+e_{1}^{2} \sin \left(2 g_{1}+u_{\mathrm{m} 1}\right)\right] e_{1} \sin \omega_{1} \sin \left(2 v_{2}+2 g_{2}\right)\right\}\right\},
\end{aligned}
$$

$$
\begin{aligned}
\frac{\mathrm{d} e_{1}^{2} f_{3}\left(e_{1}\right) \sin 2 \omega_{1}}{\mathrm{~d} v_{2}}= & A_{\mathrm{L}}\left(1+e_{2} \cos v_{2}\right)\left\{( 1 - e _ { 1 } ^ { 2 } ) ^ { 1 / 2 } \left\{\frac{6}{5} e_{1}^{2} f_{3} \cos 2 \omega_{1}\left[\left(I^{2}-\frac{1}{3}\right)+\left(1-I^{2}\right) \cos \left(2 v_{2}+2 g_{2}\right)\right]\right.\right. \\
& +\left[2 e_{1}^{2} f_{4} \cos \left(2 \omega_{1}-2 g_{1}\right)-\frac{1}{6} e_{1}^{4} f_{5} \cos \left(2 \omega_{1}+2 g_{1}\right)\right]\left[\left(1-I^{2}\right)+\left(1+I^{2}\right) \cos \left(2 v_{2}+2 g_{2}\right)\right] \\
& \left.-\left[2 e_{1}^{2} f_{4} \sin \left(2 \omega_{1}-2 g_{1}\right)+\frac{1}{6} e_{1}^{4} f_{5} \sin \left(2 \omega_{1}+2 g_{1}\right)\right] 2 I \sin \left(2 v_{2}+2 g_{2}\right)\right\} \\
& +\frac{\sin i_{\mathrm{m}} \cot i_{1}}{\left(1-e_{1}^{2}\right)^{1 / 2}} f_{3}\left\{\left[-\frac{4}{5}\left(1+\frac{3}{2} e_{1}^{2}\right) \cos u_{\mathrm{m} 1}+2 e_{1}^{2} \cos \left(2 g_{1}+u_{\mathrm{m} 1}\right)\right] e_{1}^{2} \cos 2 \omega_{1} I\left[1-\cos \left(2 v_{2}+2 g_{2}\right)\right]\right. \\
& \left.\left.-\left[\frac{4}{5}\left(1+\frac{3}{2} e_{1}^{2}\right) \sin u_{\mathrm{m} 1}+2 e_{1}^{2} \sin \left(2 g_{1}+u_{\mathrm{m} 1}\right)\right] e_{1}^{2} \cos 2 \omega_{1} \sin \left(2 v_{2}+2 g_{2}\right)\right\}\right\},
\end{aligned}
$$

$$
\begin{aligned}
\frac{\mathrm{d} e_{1}^{3} f_{6}\left(e_{1}\right) \cos 3 \omega_{1}}{\mathrm{~d} v_{2}}= & A_{\mathrm{L}}\left(1+e_{2} \cos v_{2}\right)\left\{( 1 - e _ { 1 } ^ { 2 } ) ^ { 1 / 2 } \left\{-\frac{9}{5} e_{1}^{3} f_{6} \sin 3 \omega_{1}\left[\left(I^{2}-\frac{1}{3}\right)+\left(1-I^{2}\right) \cos \left(2 v_{2}+2 g_{2}\right)\right]\right.\right. \\
& -\left[3 e_{1}^{3} f_{7} \sin \left(3 \omega_{1}-2 g_{1}\right)-\frac{3}{8} e_{1}^{5} \sin \left(3 \omega_{1}+2 g_{1}\right)\right]\left[\left(1-I^{2}\right)+\left(1+I^{2}\right) \cos \left(2 v_{2}+2 g_{2}\right)\right] \\
& \left.-\left[3 e_{1}^{3} f_{7} \cos \left(3 \omega_{1}-2 g_{1}\right)+\frac{3}{8} e_{1}^{5} \cos \left(3 \omega_{1}+2 g_{1}\right)\right] 2 I \sin \left(2 v_{2}+2 g_{2}\right)\right\} \\
& +\frac{\sin i_{\mathrm{m}} \cot i_{1}}{\left(1-e_{1}^{2}\right)^{1 / 2}} f_{6}\left\{\left[\frac{6}{5}\left(1+\frac{3}{2} e_{1}^{2}\right) \cos u_{\mathrm{m} 1}-3 e_{1}^{2} \cos \left(2 g_{1}+u_{\mathrm{m} 1}\right)\right] e_{1}^{3} \sin 3 \omega_{1} I\left[1-\cos \left(2 v_{2}+2 g_{2}\right)\right]\right. \\
& \left.\left.+\left[\frac{6}{5}\left(1+\frac{3}{2} e_{1}^{2}\right) \sin u_{\mathrm{m} 1}+3 e_{1}^{2} \sin \left(2 g_{1}+u_{\mathrm{m} 1}\right)\right] e_{1}^{3} \sin 3 \omega_{1} \sin \left(2 v_{2}+2 g_{2}\right)\right\}\right\},
\end{aligned}
$$

$$
\begin{aligned}
\frac{\mathrm{d} e_{1}^{4} f_{8}\left(e_{1}\right) \sin 4 \omega_{1}}{\mathrm{~d} v_{2}}= & A_{\mathrm{L}}\left(1+e_{2} \cos v_{2}\right)\left\{( 1 - e _ { 1 } ^ { 2 } ) ^ { 1 / 2 } \left\{\frac{12}{5} e_{1}^{4} f_{8} \cos 4 \omega_{1}\left[\left(I^{2}-\frac{1}{3}\right)+\left(1-I^{2}\right) \cos \left(2 v_{2}+2 g_{2}\right)\right]\right.\right. \\
& +\left[4 e_{1}^{4} f_{5} \cos \left(4 \omega_{1}-2 g_{1}\right)-\frac{3}{5} e_{1}^{6} \cos \left(4 \omega_{1}+2 g_{1}\right)\right]\left[\left(1-I^{2}\right)+\left(1+I^{2}\right) \cos \left(2 v_{2}+2 g_{2}\right)\right] \\
& \left.-\left[4 e_{1}^{4} f_{5} \sin \left(4 \omega_{1}-2 g_{1}\right)+\frac{3}{5} e_{1}^{6} \sin \left(4 \omega_{1}+2 g_{1}\right)\right] 2 I \sin \left(2 v_{2}+2 g_{2}\right)\right\} \\
& +\frac{\sin i_{\mathrm{m}} \cot i_{1}}{\left(1-e_{1}^{2}\right)^{1 / 2}} f_{8}\left\{\left[-\frac{8}{5}\left(1+\frac{3}{2} e_{1}^{2}\right) \cos u_{\mathrm{m} 1}+4 e_{1}^{2} \cos \left(2 g_{1}+u_{\mathrm{m} 1}\right)\right] e_{1}^{4} \cos 4 \omega_{1} I\left[1-\cos \left(2 v_{2}+2 g_{2}\right)\right]\right. \\
& \left.\left.-\left[\frac{8}{5}\left(1+\frac{3}{2} e_{1}^{2}\right) \sin u_{\mathrm{m} 1}+4 e_{1}^{2} \sin \left(2 g_{1}+u_{\mathrm{m} 1}\right)\right] e_{1}^{4} \cos 4 \omega_{1} \sin \left(2 v_{2}+2 g_{2}\right)\right\}\right\},
\end{aligned}
$$


T. Borkovits et al.: Transit timing variations in eccentric hierarchical triple exoplanetary systems. I.

$$
\begin{aligned}
\frac{\mathrm{d} e_{1}^{5} \cos 5 \omega_{1}}{\mathrm{~d} v_{2}}= & A_{\mathrm{L}}\left(1+e_{2} \cos v_{2}\right)\left\{( 1 - e _ { 1 } ^ { 2 } ) ^ { 1 / 2 } \left\{-3 e_{1}^{5} \sin 5 \omega_{1}\left[\left(I^{2}-\frac{1}{3}\right)+\left(1-I^{2}\right) \cos \left(2 v_{2}+2 g_{2}\right)\right]\right.\right. \\
& -5 e_{1}^{5} \sin \left(5 \omega_{1}-2 g_{1}\right)\left[\left(1-I^{2}\right)+\left(1+I^{2}\right) \cos \left(2 v_{2}+2 g_{2}\right)\right] \\
& \left.-5 e_{1}^{5} \cos \left(5 \omega_{1}-2 g_{1}\right) 2 I \sin \left(2 v_{2}+2 g_{2}\right)\right\} \\
& +\frac{\sin i_{\mathrm{m}} \cot i_{1}}{\left(1-e_{1}^{2}\right)^{1 / 2}}\left\{\left[2\left(1+\frac{3}{2} e_{1}^{2}\right) \cos u_{\mathrm{m} 1}-5 e_{1}^{2} \cos \left(2 g_{1}+u_{\mathrm{m} 1}\right)\right] e_{1}^{5} \sin 5 \omega_{1} I\left[1-\cos \left(2 v_{2}+2 g_{2}\right)\right]\right. \\
& \left.\left.+\left[2\left(1+\frac{3}{2} e_{1}^{2}\right) \sin u_{\mathrm{m} 1}+5 e_{1}^{2} \sin \left(2 g_{1}+u_{\mathrm{m} 1}\right)\right] e_{1}^{5} \sin 5 \omega_{1} \sin \left(2 v_{2}+2 g_{2}\right)\right\}\right\}
\end{aligned}
$$

$$
\begin{aligned}
\frac{\mathrm{d} e_{1}^{6} \sin 6 \omega_{1}}{\mathrm{~d} v_{2}}= & A_{\mathrm{L}}\left(1+e_{2} \cos v_{2}\right)\left\{( 1 - e _ { 1 } ^ { 2 } ) ^ { 1 / 2 } \left\{\frac{18}{5} e_{1}^{6} \cos 6 \omega_{1}\left[\left(I^{2}-\frac{1}{3}\right)+\left(1-I^{2}\right) \cos \left(2 v_{2}+2 g_{2}\right)\right]\right.\right. \\
& +6 e_{1}^{6} \cos \left(6 \omega_{1}-2 g_{1}\right)\left[\left(1-I^{2}\right)+\left(1+I^{2}\right) \cos \left(2 v_{2}+2 g_{2}\right)\right] \\
& \left.-6 e_{1}^{6} \sin \left(6 \omega_{1}-2 g_{1}\right) 2 I \sin \left(2 v_{2}+2 g_{2}\right)\right\} \\
& +\frac{\sin i_{\mathrm{m}} \cot i_{1}}{\left(1-e_{1}^{2}\right)^{1 / 2}} f_{8}\left\{\left[-\frac{12}{5}\left(1+\frac{3}{2} e_{1}^{2}\right) \cos u_{\mathrm{m} 1}+6 e_{1}^{2} \cos \left(2 g_{1}+u_{\mathrm{m} 1}\right)\right] e_{1}^{4} \cos 6 \omega_{1} I\left[1-\cos \left(2 v_{2}+2 g_{2}\right)\right]\right. \\
& \left.\left.-\left[\frac{12}{5}\left(1+\frac{3}{2} e_{1}^{2}\right) \sin u_{\mathrm{m} 1}+6 e_{1}^{2} \sin \left(2 g_{1}+u_{\mathrm{m} 1}\right)\right] e_{1}^{6} \cos 6 \omega_{1} \sin \left(2 v_{2}+2 g_{2}\right)\right\}\right\} .
\end{aligned}
$$

The direct terms are coming from $\left(a^{3 / 2} e^{m} \cos (n v)\right.$ and $\left.\dot{\Omega} \cos i_{1}\right)$ are as follows:

$$
\begin{aligned}
\left(\frac{\mathrm{d} e_{1} \cos v}{\mathrm{~d} v_{2}}\right)_{\mathrm{dir}}= & A_{\mathrm{L}}\left(1+e_{2} \cos v_{2}\right)\left(1-e_{1}^{2}\right)^{1 / 2}\left\{-\frac{2}{5}\left(1+\frac{1}{2} e_{1}^{2}\right)\left[\left(I^{2}-\frac{1}{3}\right)+\left(1-I^{2}\right) \cos \left(2 v_{2}+2 g_{2}\right)\right]\right. \\
& -e_{1}^{2} \cos 2 g_{1}\left[\left(1-I^{2}\right)+\left(1+I^{2}\right) \cos \left(2 v_{2}+2 g_{2}\right)\right] \\
& \left.-e_{1}^{2} \sin 2 g_{1} 2 I \sin \left(2 v_{2}+2 g_{2}\right)\right\}+\ldots,
\end{aligned}
$$

$$
\begin{aligned}
\left(\frac{\mathrm{d} e_{1}^{2} f_{3}\left(e_{1}\right) \cos 2 v}{\mathrm{~d} v_{2}}\right)_{\mathrm{dir}}= & A_{\mathrm{L}}\left(1+e_{2} \cos v_{2}\right)\left(1-e_{1}^{2}\right)^{1 / 2}\left\{\frac{3}{5} e_{1}^{2}\left(1+\frac{1}{4} e_{1}^{2}+\frac{1}{18} e_{1}^{4}\right)\left[\left(I^{2}-\frac{1}{3}\right)+\left(1-I^{2}\right) \cos \left(2 v_{2}+2 g_{2}\right)\right]\right. \\
& -\frac{1}{5} e_{1}^{2}\left(1-\frac{53}{12} e_{1}^{2}-\frac{3}{2} e_{1}^{4}\right) \cos 2 g_{1}\left[\left(1-I^{2}\right)+\left(1+I^{2}\right) \cos \left(2 v_{2}+2 g_{2}\right)\right] \\
& \left.-\frac{1}{5} e_{1}^{2}\left(1-\frac{53}{12} e_{1}^{2}-\frac{3}{2} e_{1}^{4}\right) \sin 2 g_{1} 2 I \sin \left(2 v_{2}+2 g_{2}\right)\right\}+\ldots,
\end{aligned}
$$

$$
\begin{aligned}
\left(\frac{\mathrm{d} e_{1}^{3} f_{6}\left(e_{1}\right) \cos 3 v}{\mathrm{~d} v_{2}}\right)_{\mathrm{dir}}= & A_{\mathrm{L}}\left(1+e_{2} \cos v_{2}\right)\left(1-e_{1}^{2}\right)^{1 / 2}\left\{-\frac{3}{5} e_{1}^{4}\left(1+\frac{1}{2} e_{1}^{2}\right)\left[\left(I^{2}-\frac{1}{3}\right)+\left(1-I^{2}\right) \cos \left(2 v_{2}+2 g_{2}\right)\right]\right. \\
& +\frac{1}{5} e_{1}^{2}\left(1+e_{1}^{2}-\frac{49}{16} e_{1}^{4}\right) \cos 2 g_{1}\left[\left(1-I^{2}\right)+\left(1+I^{2}\right) \cos \left(2 v_{2}+2 g_{2}\right)\right] \\
& \left.+\frac{1}{5} e_{1}^{2}\left(1+e_{1}^{2}-\frac{49}{16} e_{1}^{4}\right) \sin 2 g_{1} 2 I \sin \left(2 v_{2}+2 g_{2}\right)\right\}+\ldots,
\end{aligned}
$$

$$
\begin{aligned}
\left(\frac{\mathrm{d} e_{1}^{4} f_{8}\left(e_{1}\right) \cos 4 v}{\mathrm{~d} v_{2}}\right)_{\mathrm{dir}}= & A_{\mathrm{L}}\left(1+e_{2} \cos v_{2}\right)\left(1-e_{1}^{2}\right)^{1 / 2}\left\{\frac{1}{2} e_{1}^{6}\left[\left(I^{2}-\frac{1}{3}\right)+\left(1-I^{2}\right) \cos \left(2 v_{2}+2 g_{2}\right)\right]\right. \\
& -\frac{2}{5} e_{1}^{4}\left(1+\frac{3}{4} e_{1}^{2}\right) \cos 2 g_{1}\left[\left(1-I^{2}\right)+\left(1+I^{2}\right) \cos \left(2 v_{2}+2 g_{2}\right)\right]
\end{aligned}
$$$$
\left.-\frac{2}{5} e_{1}^{4}\left(1+\frac{3}{4} e_{1}^{2}\right) \sin 2 g_{1} 2 I \sin \left(2 v_{2}+2 g_{2}\right)\right\}+\ldots
$$

$$
\begin{aligned}
\left(\frac{\mathrm{d} e_{1}^{5} \cos 5 v}{\mathrm{~d} v_{2}}\right)_{\mathrm{dir}}= & A_{\mathrm{L}}\left(1+e_{2} \cos v_{2}\right)\left(1-e_{1}^{2}\right)^{1 / 2}\left\{\frac{1}{2} e_{1}^{6} \cos 2 g_{1}\left[\left(1-I^{2}\right)+\left(1+I^{2}\right) \cos \left(2 v_{2}+2 g_{2}\right)\right]\right. \\
& \left.+\frac{1}{2} e_{1}^{6} \sin 2 g_{1} 2 I \sin \left(2 v_{2}+2 g_{2}\right)\right\}+\ldots,
\end{aligned}
$$


$\left(\frac{\mathrm{d} e_{1}^{6} \cos 6 v}{\mathrm{~d} v_{2}}\right)_{\mathrm{dir}}=0$

where $+\ldots$ refer to those terms which come from the normal force component, and will be cancelled by equal but opposite in sign $\operatorname{direct} \dot{\Omega}$-terms. Furthermore, from

$$
\begin{aligned}
\left(\mu^{-1 / 2} \frac{\mathrm{d} a^{3 / 2}}{\mathrm{~d} v_{2}} \frac{\left(1-e_{1}^{2}\right)^{3 / 2}}{\left(1+e_{1} \cos v\right)^{2}}\right)_{\mathrm{dir}}= & \frac{P_{1}}{2 \pi} A_{\mathrm{L}}\left(1+e_{2} \cos v_{2}\right)\left(1-e_{1}^{2}\right)^{1 / 2}\left\{\frac{6}{5} e_{1}^{2}\left(1+\frac{9}{16} e_{1}^{2}+\frac{7}{16} e_{1}^{4}\right)\left[\left(I^{2}-\frac{1}{3}\right)+\left(1-I^{2}\right) \cos \left(2 v_{2}+2 g_{2}\right)\right]\right. \\
& +\frac{21}{20} e_{1}^{2}\left(1+\frac{9}{14} e_{1}^{2}+\frac{57}{112} e_{1}^{4}\right) \cos 2 g_{1}\left[\left(1-I^{2}\right)+\left(1+I^{2}\right) \cos \left(2 v_{2}+2 g_{2}\right)\right] \\
& \left.+\frac{21}{20} e_{1}^{2}\left(1+\frac{9}{14} e_{1}^{2}+\frac{57}{112} e_{1}^{4}\right) \sin 2 g_{1} 2 I \sin \left(2 v_{2}+2 g_{2}\right)\right\}
\end{aligned}
$$

and from $\dot{\Omega}$-term:

$$
\begin{aligned}
\left(\frac{\mathrm{d} \Omega}{\mathrm{d} v_{2}} \cos i_{1} \frac{\left(1-e_{1}^{2}\right)^{3 / 2}}{\left(1+e_{1} \cos v\right)^{2}}\right)_{\mathrm{dir}}= & A_{\mathrm{L}}\left(1+e_{2} \cos v_{2}\right) \frac{\sin i_{\mathrm{m}} \cot i_{1}}{\left(1-e_{1}^{2}\right)^{1 / 2}}\left\{\left[-\frac{2}{5}\left(1+\frac{3}{2} e_{1}^{2}\right) \cos u_{\mathrm{m}_{1}}+e_{1}^{2} \cos \left(u_{\mathrm{m} 1}+2 g_{1}\right)\right] I\left[1-\cos \left(2 v_{2}+2 g_{2}\right)\right]\right. \\
& {\left.\left[-\frac{2}{5}\left(1+\frac{3}{2} e_{1}^{2}\right) \sin u_{\mathrm{m}_{1}}+e_{1}^{2} \sin \left(u_{\mathrm{m} 1}+2 g_{1}\right)\right] \sin \left(2 v_{2}+2 g_{2}\right)\right\}-\ldots }
\end{aligned}
$$

By the use of equations above, and integrating for $v_{2}$, finally we get:

$$
\begin{aligned}
(O-C)_{v_{2}}= & \frac{P_{1}}{2 \pi} A_{\mathrm{L}}\left\{( 1 - e _ { 1 } ^ { 2 } ) ^ { 1 / 2 } \left\{\left[\frac{4}{5} f_{1}\left(e_{1}\right)+\frac{6}{5} K_{1}\left(e_{1}, \omega_{1}\right)\right]\left[\left(I^{2}-\frac{1}{3}\right) \mathcal{M}+\frac{1}{2}\left(1-I^{2}\right) \mathcal{S}\left(2 v_{2}+2 g_{2}\right)\right]\right.\right. \\
& +\left[\frac{51}{20} e_{1}^{2} f_{2}\left(e_{1}\right) \cos 2 g_{1}+2 K_{2}\left(e_{1}, \omega_{1}, g_{1}\right)+\frac{1}{8} e_{1}^{2} K_{4}\left(e_{1}, \omega_{1}, g_{1}\right)\right]\left[\left(1-I^{2}\right) \mathcal{M}+\frac{1}{2}\left(1+I^{2}\right) \mathcal{S}\left(2 v_{2}+2 g_{2}\right)\right] \\
& \left.-\frac{1}{2}\left[\frac{51}{20} e_{1}^{2} f_{2}\left(e_{1}\right) \sin 2 g_{1}+2 K_{3}\left(e_{1}, \omega_{1}, g_{1}\right)+\frac{1}{8} e_{1}^{2} K_{5}\left(e_{1}, \omega_{1}, g_{1}\right)\right] 2 I C\left(2 v_{2}+2 g_{2}\right)\right\} \\
& +\frac{\sin i_{\mathrm{m}} \cot i_{1}}{\left(1-e_{1}^{2}\right)^{1 / 2}}\left\{\left[-\frac{2}{5}\left(1+\frac{3}{2} e_{1}^{2}\right) \cos u_{\mathrm{m} 1}+e_{1}^{2} \cos \left(2 g_{1}+u_{\mathrm{m} 1)}\right)\right]\left[1+2 K_{1}\left(e_{1}, \omega_{1}\right)\right] I\left[\mathcal{M}-\frac{1}{2} \mathcal{S}\left(2 v_{2}+2 g_{2}\right)\right]\right. \\
& \left.\left.+\frac{1}{2}\left[\frac{2}{5}\left(1+\frac{3}{2} e_{1}^{2}\right) \sin u_{\mathrm{m} 1}+e_{1}^{2} \sin \left(2 g_{1}+u_{\mathrm{m} 1)}\right)\right]\left[1+2 K_{1}\left(e_{1}, \omega_{1}\right)\right] C\left(2 v_{2}+2 g_{2}\right)\right\}\right\},
\end{aligned}
$$

where

$$
\begin{aligned}
K_{1}\left(e_{1}, \omega_{1}\right)= & \mp e_{1} \sin \omega_{1}+\frac{3}{4} e_{1}^{2} f_{3} \cos 2 \omega_{1} \pm \frac{1}{2} e_{1}^{3} f_{6} \sin 3 \omega_{1}-\frac{5}{16} e_{1}^{4} f_{8} \cos 4 \omega_{1} \mp \frac{3}{16} e_{1}^{5} \sin 5 \omega_{1}+\frac{7}{64} e_{1}^{6} \cos 6 \omega_{1}, \\
K_{2}\left(e_{1}, \omega_{1}, g_{1}\right)= & \mp e_{1} \sin \left(\omega_{1}-2 g_{1}\right)+\frac{3}{4} e_{1}^{2} f_{4} \cos \left(2 \omega_{1}-2 g_{1}\right) \pm \frac{1}{2} e_{1}^{3} f_{7} \sin \left(3 \omega_{1}-2 g_{1}\right)-\frac{5}{16} e_{1}^{4} f_{5} \cos \left(4 \omega_{1}-2 g_{1}\right) \\
& \mp \frac{3}{16} e_{1}^{5} \sin \left(5 \omega_{1}-2 g_{1}\right)+\frac{7}{64} e_{1}^{6} \cos \left(6 \omega_{1}-2 g_{1}\right),
\end{aligned}
$$

$K_{3}\left(e_{1}, \omega_{1}, g_{1}\right)=\mp e_{1} \cos \left(\omega_{1}-2 g_{1}\right)-\frac{3}{4} e_{1}^{2} f_{4} \sin \left(2 \omega_{1}-2 g_{1}\right) \pm \frac{1}{2} e_{1}^{3} f_{7} \cos \left(3 \omega_{1}-2 g_{1}\right)+\frac{5}{16} e_{1}^{4} f_{5} \sin \left(4 \omega_{1}-2 g_{1}\right)$

$$
\mp \frac{3}{16} e_{1}^{5} \cos \left(5 \omega_{1}-2 g_{1}\right)-\frac{7}{64} e_{1}^{6} \sin \left(6 \omega_{1}-2 g_{1}\right)
$$

$K_{4}\left(e_{1}, \omega_{1}, g_{1}\right)=-e_{1}^{2} f_{5} \cos \left(2 \omega_{1}+2 g_{1}\right) \mp e_{1}^{3} \sin \left(3 \omega_{1}+2 g_{1}\right)+\frac{3}{4} e_{1}^{4} \cos \left(4 \omega_{1}+2 g_{1}\right)$,

$K_{5}\left(e_{1}, \omega_{1}, g_{1}\right)=-e_{1}^{2} f_{5} \sin \left(2 \omega_{1}+2 g_{1}\right) \pm e_{1}^{3} \cos \left(3 \omega_{1}+2 g_{1}\right)+\frac{3}{4} e_{1}^{4} \sin \left(4 \omega_{1}+2 g_{1}\right)$, 
T. Borkovits et al.: Transit timing variations in eccentric hierarchical triple exoplanetary systems. I.

and

$f_{1}=1+\frac{25}{8} e_{1}^{2}+\frac{15}{8} e_{1}^{4}+\frac{95}{64} e_{1}^{6}$

$f_{2}=1+\frac{31}{51} e_{1}^{2}+\frac{23}{48} e_{1}^{4}$,

$f_{3}=1+\frac{1}{6} e_{1}^{2}+\frac{1}{16} e_{1}^{4}$,

$f_{4}=1+\frac{1}{4} e_{1}^{2}+\frac{1}{8} e_{1}^{4}$

$f_{5}=1+\frac{3}{4} e_{1}^{2}$

$f_{6}=1+\frac{3}{8} e_{1}^{2}$,

$f_{7}=1+\frac{1}{2} e_{1}^{2}$,

$f_{8}=1+\frac{3}{5} e_{1}^{2}$. 